\title{
Mogens Flensted-JEnsen
}

KIYOSATO OKAMOTO

\section{An explicit construction of the K-finite vectors in the discrete series for an isotropic semisimple symmetric space}

Mémoires de la S. M. F. $2^{e}$ série, tome 15 (1984), p. 157-199

<http://www.numdam.org/item?id=MSMF_1984_2_15_157_0>

C Mémoires de la S. M. F., 1984, tous droits réservés.

L'accès aux archives de la revue « Mémoires de la S. M. F. » (http://smf. emath.fr/Publications/Memoires/Presentation.html) implique l'accord avec les conditions générales d'utilisation (http://www.numdam.org/conditions). Toute utilisation commerciale ou impression systématique est constitutive d'une infraction pénale. Toute copie ou impression de ce fichier doit contenir la présente mention de copyright.

\section{Numdam}


Societe Mathematique de Prance

2e série, Mémoire n॰ 15, 1984, p. 157-199

AN EXPLICIT CONSTEUCTION OF THE $K$ - FINITE VECTORS IN THE DISCRETE SERIES FOR AN ISOTROPIC SEMISIMPLE SMMETRIC SPACE.

BY

mbgens flensted - Jensen* and Kiyosato OKamoto*

\footnotetext{
- Both authors partially supported by the
} Danish Natural Science Research Council. 


\section{FLENSTED-JENSEN and $\mathrm{K}$. OKNYTO}

$\S 1$. Introduction.

In [18] Strichartz stated an explicit formula describing all $O(n) \times O(N)$ - finite functions in any $O(n, N)$ - invariant, closed and irreducible subspace of $L^{2}(O(n, N) / O(n, N-1))$. From a grouptheoretical point of view the formula is not so transparent since its formulation uses an explicit realization of $O(n, N) / O(n, N-1)$ as a hyperbolic space in $\mathbb{R}^{\mathrm{n}+\mathrm{N}}$.

In this paper we suggest a formula, which may do the same for the general semisimple symmetric space $G / H, i . e$. describe the $K$-finite functions in the irreducible representations of $G$ in $L^{2}(G / H)$. The socalled discrete series for $G / H$. For the general case we can only state a few necessary and a few sufficient conditions for our formula to give a $K$-finite function in a discrete series for $G / H$. However for the isotropic spaces $G / H$ the formula suffices to describe all the $\mathrm{K}$-types of all the discrete series for $\mathrm{G} / \mathrm{H}$.

By the classification, cf. Wolf [22] and Berger [1]. The pseudoRiemannian, nonRiemannian isotropic spaces are all symmetric. Up to coverings they are the classical real-, complex- and quarternionic projective hyperbolic spaces.

$$
\begin{aligned}
& \operatorname{so}_{e}(p, q+1) / S(O(p, q) \times O(1)) \\
& S U(p, q+1) / S(U(p, q) \times U(1)) \\
& S p(p, q+1) / S p(p, q) \times S p(1)
\end{aligned}
$$

for $p \geq 1$ and $q \geq 1$, and one exceptional case

$$
F_{4(-20)} / \operatorname{Spin}(1,8)
$$

This last space may in some sense be thought of as the projective hyperbolic space over the Cayley numbers with $p=q=1$. We are not interested in the Riemannian isotropic spaces, since they have no discrete series.

our formula, when explicitly computed for a real hyperbolic space, gives a formula very similar to Strichartz', but not completely identical to 1t. This difference between the two formulas may contain some nontrivial relations between formulas for special functions.

Our interest in the problem came from a discussion of the paper Flensted-Jensen [3]. In that paper it is shown that if rank $G / H=r a n k ~ K / K \cap H$ then discrete series do 


\section{Discrete series}

exist. The existence is shown by construction of $\mathrm{K}$-finite elements in the corresponding subspaces of $L^{2}(G / H)$. In section 8 of [loc. cit.] it was shown by looking at the nonRiemannian isotropic spaces that the construction did not exhaust the discrete series for these spaces, at least for $q$ large compared to $p$. It is our hope that the present study of these exceptional discrete series for the isotropic spaces may give some hints of how to solve the general problem of construction of all discrete series for $G / H$.

A recent preprint of Oshima-Matsuki [14] contains very much information on "where to expect" these exceptionel discrete series in general. However the general problem of actual construction of $\mathrm{K}$-finite elements in each representation space seems still open.

We get two offspins of our result: In Section 3 we find a new proof of the minimality of the K-types used in [3] to construct the discrete series for $G / H$. This proof is simpler than the proof by scinlichtkrul] [16] and is also valid for the universal covering space $G \sim / H$, which was not covered by [16], because of the use of results from Vogan-Speh [21]. In Eksample 4.8 the computations show that there are examples where a minimal $K$-type of a discrete series for $G / H$ does not have a $\mathrm{k} \cap \mathrm{H}-\mathrm{fixed}$ vector. In contrast to what for a long time was the belief of the first auther, cf. [4]. Also non-uniqueness of minimal K-types occurs.

The content of the present paper is as follows: In Section 2 we introduce the necessary notation and prerequisits. In section 3 we introduce and discuss our proposed integral formula. In section 4 we turn to the case of rank one and in particular to the isotropic spaces. For these our formula gives the complete answer. In Section 5 we compute explicitly the formulas optained in section 4 .

We want to thank Plesner Jakobsen and Schlichtkrull for many fruitfull discussions concerning the content of the present paper. 


\section{FLENSTED-JENSEN and $\mathrm{R}$. OKAMOTO}

\section{$\S 2$. Notation and preliminaries.}

Let $G$ be a connected, linear semisimple lie group contained as a real form in a complex, simply connected Lie Group $G_{\mathcal{G}}$. Let $\tau$ be an involution of $G$ and let $H=G_{e}^{\top}$ be the identity component of the fixpoints for $\tau$. Let $X^{\sim}=G^{\sim} / H$ be the universal covering space of $X=G / H^{1)}$. Every connected, simply connected semisimple symmetric space is of the form $X^{\sim}=G^{\sim} / H$. For more detailes see Berger [1], Loos [12] or Flensted-Jensen [4].

If $x^{\sim}$ is irreducible, then $x$ is one of the following three types

(I) The compact type if $G$ is compact.

(II) The noncompact type if $\mathrm{H}$ is compact and $\mathrm{G}$ is noncompact.

(III) The nonRiemannian type if $\mathrm{H}$ in noncompact.

The Killing form induces an invariant metric on $x$ and on $x^{\sim}$. In case (I) and (II) the metric is Riemannian. In case (III) it is pseudoRiemanninan.

Up to H-conjugacy there is a unique maximal compact subgroup $K$ of $G$, such that $\tau(K)=K$. Let $\sigma$ be the Cartan involution related to $K$. Then $\sigma \tau=\tau \sigma$. Notice that for $x^{\sim}$ irreducible the three types can be characterized by: (I) $G=K$, . (II) $\mathrm{H}=\mathrm{K}$ and (III) $\mathrm{G} \neq \mathrm{K}$ and $\mathrm{H} \neq \mathrm{K}$.

Examples. (a). A connected semisimple Lie group $G_{1}$ may be considered as a symmetric space. Let $d\left(G_{1}\right)$ be the diagonal subgroup in $G_{1} \times G_{1}$, then $G_{1} \times G_{1} / d\left(G_{1}\right)$ is a symmetric space, which as a manifold is isomorphic to $G_{1}$. It is of type (I) if $G_{1}$ is compact. Otherwise it is of type (III).

(b). The hyperbolic spaces mentioned in Section 1 is of type (I) if $p=0$, type (II) if $p \geq 1$ and $q=0$ and type (III) if $p \geq 1$ and $q \geq 1$.

The Riemannian symmetric spaces are well studied, cf. f.ex. Helgason [6], [8] and [9]. Our main concern in this paper is the nonRiemannian spaces. However as described below we shall make extensive use of a Riemannian symmetric space $\mathrm{G}^{\circ} / \mathrm{H}^{\circ}$ "dual" to the nonRiemannian space $G / H$.

1) $\mathrm{G}^{\sim}$ is chosen such that the covering map of $\mathrm{G}^{\sim}$ onto $G$ is an isomorphism between the analytic subgroups corresponding to $h$, the Lie algebra of $H$. 
Definitions. (i) A discrete series representation $\pi$ for $X=G / H$ is (the unitary equivalence class of) the representation of $G$ on an invariant, irreducible subspace $V \neq 0$ of $L^{2}(x)$.

(ii) $v_{k}$ is the subset of $v$ consisting of the $k$-finite vectors in $v$. Then $v_{K} \subset L^{2}(x) \cap c^{\infty}(x)$.

(iii) $v_{\min }$ is the union of the isotypic components of the minimal $K$-types in $V_{K}$, (in the sense of Vogan [20]). Any $\psi \in V_{M i n}, \psi \neq 0$ is called a minimal spherical function ${ }^{1)}$ for $\pi$.

(iv) For $\mathrm{X}^{\sim}=\mathrm{G}^{\sim} / \mathrm{H}$ a discrete series (or more precisely a relative discrete series) is defined as under (i), but modulo a unitary action of the center of $G^{\sim}$.

(The modifications needed in this connection are rather obvious. We shall not in the following be completely consistent in always pointing out the necessary reformulations in order to incorporate $\mathrm{G} / \mathrm{H}$ into the treatment.)

Let $g_{\uparrow}$ be the complex Lie algebra of $G_{\uparrow}$. Let $g, h$ and $k$ be the real subalgebras corresponding to $G, H$ and $K$. We denote again by $\tau$ and $\sigma$ the differentials of $\tau$ and $\sigma$ and their holomorphic extensions to $g_{\downarrow}$. Since $\tau$ and $\sigma$ commute we may decompose $g$ according to +1 and -1 eigenspaces for $\tau$ and $\sigma$ in the following way:

$$
g=h+q=k+p=h n k+h n p+q n k+q n p .
$$

Inside $g_{\downarrow}$ we now define the real subalgebras $g^{\circ}, h^{\circ}$ and $k^{\circ}$ "dial" to $g, h$ and $k$ by

$$
\begin{aligned}
& g^{\circ}=h n k+i(h \cap p+q n k)+q n p . \\
& h^{\circ}=h_{\Phi} n g^{\circ} \text { and } k^{\circ}=k_{\Phi} n g^{\circ} .
\end{aligned}
$$

where $i=\sqrt{-1}$

Let $\mathrm{G}^{\circ}, \mathrm{H}^{\circ}$ and $\mathrm{K}^{\circ}$ be the corresponding analytic subgroups of $\mathrm{G}_{\mathbb{\$}}$. Notice that $\mathrm{H}^{\circ}$ is maximal compact in $\mathrm{G}^{\circ}$, whereas $\mathrm{K}^{\circ}$ is (in general) noncompact.

Definition. We shall call the Riemannian symmetric space $G^{\circ} / H^{\circ}$ with the associated symmetric subgroup $K^{\circ}$ for the dual to $G / H$.

Notice that $(G / K, H)$ is dual to $G^{0} / K^{\circ}$.

1) This definition is slightly more general than the one used in [4]. 


\section{FLENSTED-JENSEN and $\mathbf{K}$. OKAMOTO}

Examples. (a). Let $G_{1}$ be a connected, semisimple Lie group (with finite center) and $K_{1}$ a maximal compact subgroup. Let $G_{1 \notin}$ and $K_{1 \notin}$ be the corresponding complex groups and let $U_{1}$ be a compact real form of $G_{1 \notin}$. The dual of $G_{1}, i . e$. of $G_{1} \times G_{1} / d\left(G_{1}\right)$ is then $\left(G_{1 \Phi} / U_{1}, K_{1}\right)$.

(b). The dual of a hyperbolic space is obtained by exchanging the index pair $(p, q)$ with $(p+q, 0)$, and the corresponding subgroup $k^{\circ}$ is in the respective cases given by $S O(p) \times S O_{e}(q, 1), S(U(p) \times U(q, 1)), S p(p) \times S p(q, 1)$ and $\operatorname{spin}(8,1)$.

Let $C_{K}^{\infty}(G / H), C_{K \sim}^{\infty}\left(G^{\sim} / H\right)$ and $C_{K}^{\infty}\left(G^{0} / H^{\circ}\right)$ denote respectively the $K-, K^{\sim}$ and $K^{0}$ - finite functions in $C^{\infty}(G / H), C^{\infty}\left(G^{\sim} / H\right)$ and $C^{\infty}\left(G^{0} / H^{0}\right)$. Let $U\left(g_{\mathbb{G}}\right)$ denote the universal enveloping algebra of $g_{\mathbb{T}}$ and let $u\left(g_{\mathbb{T}}\right)^{h}$ and $u\left(g_{\mathbb{T}}\right)^{k}$ denote the centralizer of respectively $h_{\mathbb{f}}$ and $k_{\mathbb{F}}$. Then $u\left(g_{\mathbb{q}}\right)$ is naturally identified with the algebra of right invariant differential operators on $G, G^{\sim}$ or $G^{\circ}$, and thus also defines differential operators on $\mathrm{G} / \mathrm{H}, \mathrm{G}^{\sim} / \mathrm{H}$ or $\mathrm{G}^{0} / \mathrm{H}^{\circ}$. Clearly $u\left(g_{\mathbb{T}}\right)$ leaves $\left.c_{K}^{\infty} / G / H\right), c_{K}^{\infty}\left(G^{\sim} / H\right)$ and $C_{K} \circ\left(G^{\circ} / H^{\circ}\right)$ invariant. Similarly $u\left(g_{\mathbb{C}}\right)^{h}$ is, modulo a certain kernel, naturally identified with the algebra of invariant differential operators on $G / H, G \sim / H$ or $G^{\circ} / H^{\circ}$.

Duality Theorem. ([3]). There is a unique isomorphism $n: f \rightarrow f^{\circ}$ between the $u\left(g_{\mathbb{C}}\right) \times U\left(g_{\mathbb{C}}\right)^{h}-$ modules $c_{K}^{\infty} \sim(G / H)$ and $c_{K}^{\infty}\left(G^{\circ} / H^{\circ}\right)$ such that

$$
f(x H)=f^{\circ}\left(x H^{\circ}\right)
$$

for each $x$ in the identity component ${ }^{1)} G_{0}$ of $\mathrm{GnG}^{\circ}$.

Definition. A Cartan subspace $a$ for $G / H$ is a maximal Abelian subspace of $q$. consisting of semisimple elements. Every Cartan subspace is H-conjugate to a o-invariant one. A o-invariant Cartan subspace is called fundamental, respectively compact, if $a$ lik is maximal Abelian in $q$ nk, respectively if $a \subset q n k$. The rank of $G / H$ is the dimension of any Cartan subspace for $G / H$.

1) Here is a slight abuse of notation. It is used that the covering map of $\mathrm{G}^{\sim}$ onto $G$ is an isomorphism between the analytic subgroups corresponding to $g_{0}=g g^{\circ}$. 
Theorem 2.1. There exist discrete series for $G / H$ if and only if $G / H$ has a compact Cartan subspaces or equivalently if and only if

(2.1) $\operatorname{rank}(G / H)=\operatorname{rank}(K / N / H)$.

The sufficiency of (2.1) is proved in [3] by a simple construction of elements in $C_{K}^{\infty}(G / H) \cap L^{2}(G / H)$ generating irreducible subspaces of $L^{2}(G / H)$. We shall return to this below. The necessity of $(2.1)$ is one of the results in Oshima-Matsuki [14].

From now on we assume the rank condition (2.1) to be satisfied. Fix a compact Cartan subspace $a=i t$ and a positive system $\Delta_{c}^{+}$of restricted ${ }^{1)}$ roots for $\Delta_{c}=\Delta\left(k^{\circ}, t\right)$. Let $\Delta=\Delta\left(g^{0}, t\right)$ and let $w_{c}$ and $w$ denote the respective Weylgroups.

Let $\lambda \in t^{*}$ be such that

(2.2) $\langle\lambda, \alpha\rangle>0$ for each $\alpha \in \Delta_{c}^{+}$and

(2.3) $\langle\lambda, \alpha\rangle \neq 0$ for each $\alpha \in \Delta$.

We can then choose ${ }^{21}$ a positive system $\Delta^{+}=\Delta_{\lambda}^{+}$such that

$$
\langle\lambda, \alpha\rangle>0 \text { for each } a \in \Delta^{+} \text {. }
$$

Let $\rho=\rho_{\lambda}$, respectively $\rho_{c}$, be half the sum counted with multiplicity of the roots in $\Delta^{+}$and $\Delta_{c}^{+}$.

1) Henceforth we shall leave out the word "restricted" .

2) The number of such choices as $\lambda$ varies is the same as the number of cosets in $w_{c} \backslash w$. 
The choice of $\Delta^{+}$defines compatible Iwasawa decompositions:

$$
g^{\circ}=h^{0}+t+n^{0} \text { and } k^{0}=(h \cap k)+t+n_{c}
$$

where $n_{c}=n^{\circ} n k^{\circ}$, and

$$
G^{\circ}=H^{\circ} \mathrm{TN}^{\circ} \text { and } \mathrm{K}^{\circ}=(\mathrm{K} / \mathrm{H}) \mathrm{TN}_{\mathrm{C}}
$$

For $x \in G^{\circ}$ we write correspondingly

$$
x=k(x) a(x) n(x) \text {. }
$$

Define also the Iwasawa projection $H: G_{0}^{0} \rightarrow t$ by $H(x)=\log (a(x))$ or

$$
x \in H^{\circ} \exp (H(x)) N^{\circ}, \quad x \in G^{\circ}
$$

We use this to define

$$
\psi_{\lambda}^{0}(x)=\int_{K \cap H} e^{\left\langle-\lambda-\rho, H\left(x^{-1} k\right)\right\rangle} d k, \quad x \in G^{\circ} .
$$

The function $\psi_{\lambda}^{0}$ is an eigenfunction of each $u \in U\left(g_{\mathbb{\Phi}}\right)^{h}$, cf. Helgason [6] page 94. Let $x_{\lambda}$ denote the corresponding eigenvalue homomorphism.

Choose a maximal Abelian subspace $b$ in $p h q$ and define the following centralizers:

$$
M^{0}=\left(H^{\circ}\right)^{t}, M_{t}=(K \cap H)^{t} \text { and } M_{b}=(K \cap H)^{b} \text {. }
$$

Furthermore define $\Delta_{c}^{-}=-\Delta_{c}^{+}, \Delta^{-}=-\Delta^{+}, n^{\circ}=\tau\left(n^{\circ}\right), n_{c}=\tau\left(n_{c}\right)$ and $n_{n}=\tau\left(n^{\circ} n p^{\circ}\right)$, and notice that $n_{n}$ is not necessarily a subalgebra.

Let $m^{0}, m_{t}$ and $m_{b}$ be the Lie algebras of respectively $M^{0}, M_{t}$ and $M_{b}$. Extend $t$ to a Cartan subalgebra $t^{\sim}$ of $k^{0}$ such that $t^{\sim}=1 t_{1}+t$. where it $\subset m_{t}$. Let $\tilde{\Delta}_{c}=\Delta\left(k_{\Phi}, \tilde{t}_{\Phi}\right)$ and $\tilde{\Delta}=\Delta\left(g_{\mathbb{q}}, \tilde{t}_{\Phi^{\prime}}\right)$ and choose compatible systems $\Delta_{c}^{+}$and $\Delta^{\sim^{c}}$ compatible with $\Delta_{c}^{+}$and $\Delta^{+}$. Let $\Delta_{\mathrm{m}}^{+}$be the corresponding positive roots for $t_{1}$ in $\left(m_{t}\right)_{t}$. Let as usual $\tilde{\Delta}_{c}=-\Delta_{c}^{m}, \tilde{\Delta}^{-}=-\Delta^{-}$ and $\Delta_{m}^{-}=-\Delta_{m}^{+}$. We embed $t^{*}$ into $\left(t_{\mathbb{t}}^{*}\right.$ in the canonical way, i.e. such that $\left\langle t^{*}, t_{1}\right\rangle=0$. 


\section{Discrete series}

The irreducible finite dimensional representations of $k_{\downarrow}$, and thus of the universal covering groups of $K^{\sim}$ or $k^{0}$, are parametrized by the dominant weights. We shall always when nothing else is mentioned mean dominant w.r.t. $\Delta_{c} \cdot$ So in particular the representation $E_{-\mu}$ with dominant weight $-\mu$ is the contragredient representation $E_{\mu}^{v}$ to the representation $E_{\mu}$ of $\tilde{\Delta}_{\mathbf{C}}^{+}$- dominant weight $\mu$. Notice, cf. Helgason [6], that if $\mu \in\left(\tilde{t}_{\uparrow}\right)$ then $-\mu$ is a dominant weight for a representation having a nontrivial $\mathrm{k} n \mathrm{H}-\mathrm{fixed}$ vector if and only if $\mu \in t^{\star}$ and

$$
\frac{\langle\mu, \alpha\rangle}{\langle\alpha, \alpha\rangle} \in \mathbb{z}^{+} \quad \text { for each } \alpha \in \Delta_{c}^{+}
$$

Let $n^{\sim}$ and $\tilde{n}$ be the sum of the rootspaces ${ }^{1)}$ in $g_{\star}$ for respectively $\Delta^{+}$ and $\tilde{\Delta}^{-}$. Then we have $\tilde{n}_{n}^{0}=n^{\circ}$ and similarly for $\vec{n}_{n}$. Let $\tilde{n}_{c}$ and $\vec{n}_{c}$ be the sum of the rootspaces in $k_{\mathbb{}}$ for respectively $\Delta_{c}$ and $\tilde{\Delta_{c}}$. Again we have $\tilde{n}_{c} n^{\circ}=n_{c}$ and similarly for $\tilde{n}_{c}$.

Let $\rho_{m}, \rho_{c}^{\sim}$ and $\rho^{\sim}$ be half the sum of the roots ${ }^{1)}$ respectively in $\Delta_{m}^{+}, \Delta_{c}^{+}$ and $\Delta^{+}$counted with multiplicity.

Let $E_{\lambda}^{0}, E_{\lambda_{\infty}}$ and $E_{\lambda}$ denote the eigenspaces of $U\left(g_{\mathbb{\Psi}}\right)^{h}$ respectively in $\mathrm{C}^{\infty}\left(\mathrm{G}^{\circ} / \mathrm{H}^{\hat{0}}\right), \mathrm{C}^{\infty}(\mathrm{G} / \mathrm{H})$ and $\mathrm{C}^{\infty}(\mathrm{G} / \mathrm{H})$ corresponding to $\mathrm{x}_{\lambda}$. By the Duality Theorem the $K^{\circ}$-finite elements in $E_{\lambda}^{0}$ is identified with the $K^{\sim}$-finite elements in $\tilde{E_{\lambda}}$. Let $L_{\lambda}^{2}(G / H)$ denote the intersection of $E_{\lambda}^{\sim}$ with the relevant $L^{2}-s p a c e$, (ci. the definition of relative discrete series).

Theorem 2.2. Let the notation be as above and let $\mu_{\lambda}=\lambda+\rho-2 \rho c$. The function $\psi_{\lambda}^{0}$ is $k^{\circ}-f i n i t e$ and generates an irreducible representation of $k^{\circ}$ with dominant weight $-\mu_{\lambda}$ if

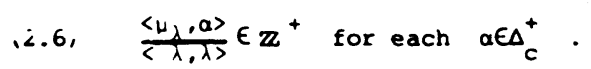

Furthermore, assuming (2.6), the dual function $\psi_{\lambda}=n^{-1}\left(\psi_{\lambda}^{0}\right)$ belongs to $L_{\lambda}^{2}(G / H)$. It is the (up to scalars) unique $\mathrm{k}$ H-fixed minimal spherical function for a discrete series representation $T_{\lambda}$ for $G / H$. $\left(T_{\lambda}\right.$ is a discrete series for $G / H$ if $-\omega_{\lambda}$ is a dominant weight for $k$ and not only for $\kappa^{\sim}$.)

1) Notice that $\Delta^{\sim}$ is not necessarily a rootsystem in the usual sense, but only the set of non-zero restrictions to $t \sim$ of a rootsystem for a cartan subalgebra in $g_{t}$. 


\section{FLENSTED-JENSEN and R. OKAMOTO}

The $k^{\circ}$-finiteness and the fact that for sufficiently large $\lambda$ s $\psi_{\lambda}$ generates a discrete series for $G^{\sim / H}$ was proved in [3]. That this holds for each $\lambda$ satisfying $(2.2),(2.3)$ and $(2.6)$ is a result of Oshima's, see Oshima-Matsuki [14] and Schlichtkrull [17]. That the representation of $\boldsymbol{K}^{\sim}$ generated by $\psi_{\lambda}$ is the unique minimal $\mathrm{K}^{\sim}$-type was first proved by Schlichtkrull [16] for the linear case, i.e. provided $-\mu_{\lambda}$ is a dominant weight for $k$ and not only for $\boldsymbol{K}^{\sim}$. In Corollary 3.6 we give another proof which holds in general.

Let now $\lambda \cdot \in t^{\star}$. Oshima and Matsuki show in [14] ${ }^{1)}$, that if $\lambda^{\prime}$ is "sufficiently regular", then $I_{\lambda^{\prime}}^{2}(G / H)$ is the direct sum of the discrete series $T_{\lambda}$, where $\lambda$ runs through the set of all $W$-translates of $\lambda^{\prime}$, which satisfies $(2.2),(2.3)$ and $(2.6)^{1)}$. Conversely they show that if $L_{\lambda^{\prime}}^{2}(G / H) \neq\{0\}$ then at least one w-translate $\lambda$ of $\lambda^{\prime}$ satisfies $(2.2),(2.3)$ and the following two conditions weaker than $(2.6):$

(2.7) $\quad \frac{\langle\mu}{\langle\alpha} \lambda \frac{, \alpha\rangle}{, \alpha\rangle} \in \mathbb{Z}$ for each $\alpha \in \Delta_{c}^{+}$

(2.8) $\langle\lambda-0, B\rangle \geq 0$ for each simple $B \in \Delta^{+}$satisfying $g_{B}^{0} \subset k^{\circ}$.

In many cases the results of Oshima and Matsuki leads to the conclusion, that the $T_{\lambda}$ 's constructed in Theorem 2.2 exhaust the discrete series for $G / H$. However in general there are two questions left open:

$1^{\circ}$. Construct for each $\lambda$ satisfying $(2.2),(2.3),(2.7)$ and $(2.8)$ but not (2.6) a discrete series representation $T_{\lambda}$ for $G \sim / H$.

$2^{\circ}$. Does the $T_{\lambda}^{\prime}$ s corresponding to all $\lambda^{\prime s}$ satisfying $(2.2),(2.3),(2.7)$ and (2.8) exhaust the discrete series for $\mathrm{G} / \mathrm{H}$.

The main problem in $1^{\circ}$ is that the function $\psi_{\lambda}^{0}$ is not $x^{\circ}$-finite. The main problem in $2^{\circ}$ besides the extension of the results in [14] to the nonlinear case is, when $\lambda$ ' is not "sufficiently regular", whether $L_{\lambda^{\prime}}^{2}(G / H)$ could contain other irreducible invariant subspaces than those coming from the $T_{\lambda}^{\prime}{ }^{\prime}$, with $\lambda \in W \cdot \lambda^{\prime}$.

1) Only the linear case is treated in [14]. Probably the results are true also for G $/ \mathbf{H}$. 
Our primary concern in this paper is question $1^{\circ}$. We should like to solve it by a simple formula similar to (2.5) giving a minimal spherical function for the unknown $T_{\lambda}$. In Section 3 we shall discuss a formula similar to $(2.5)$, which may give the answer to $1^{\circ}$. However we can only prove rather little in general. Therefore we turn in Section 4 to the case of $\operatorname{rank}(G / H)=1$. In which case we can solve question $1^{\circ}$ completely. Actually for the isotropic spaces the solution of question $2^{\circ}$ also follows and furthermore every $\mathrm{K}^{\sim}$-type of every discrete series for $\mathrm{G}^{\sim} / \mathrm{H}$ is constructed.

The results from Oshima-Matsuki [14] are mentioned here mostly to describe the motivation for our study, and to place our results in proper perspective. In fact the only places where our results are based on [14] are in the statements that the $K^{2}$-finite functions we construct in $E_{\lambda}$ are actually in $L_{\lambda}^{2}(G / H)$. This result from [14] does not depend on the linearity of $G$. The use we make of [14] is parallelle to what is mentioned above regarding the proof of Theorem 2.2. In the applications in Section 4 to the rank one case we can instead of [14] use the explicit computations in section 5 to prove that the functions are square integrable.

5 3. Integral formulas and dominant weight vectors for the K-types.

Let the notation be as in Section 2. When $\lambda$ satisfies the conditions of Theorem 2.2 then $\psi_{\lambda}$ is the $\mathrm{k} \cap \mathrm{H}$-fixed vector in the $\tilde{k}^{\sim}$-type with dominant weight $-\mu_{\lambda}$ of $T_{\lambda}$. The following formula is giving to us the corresponding dominant weight vector

(3.1) $\xi_{\lambda}^{\circ}(x)=\int_{\bar{N}_{C}} e^{\left\langle-\lambda-0, H\left(x^{-1} \bar{n}\right)\right\rangle} d \bar{n}, \quad x \in G^{0}$.

Definition. We shall often use the following short, convenient notation: Let $f \in C^{\infty}(G), x, y \in G$ and $u \in U\left(g_{d}\right)$ then we write

(3.2) $\quad f(x u y)=\left(u f^{y}\right)(x)$,

where $u$ is used as a left-invariant differential operator on $G$ and $f^{y}$ is defined by $f^{y}(g)=f(g y)$.

For example if $x \in g$ we get 


\section{FLENSTED-JENSEN and $\mathrm{K}$. OKAMOTO}

$$
f(x x y)=\left.\frac{d}{d t} f(x \exp (t x) y)\right|_{t=0,}
$$

or if $u \rightarrow u^{v}$ is the canonical antiautomorphism of $u\left(g_{\Phi}\right)$, then $u$ as a rightinvariant operator applied to $f i$ given by $u f(x)=f\left(u^{v} x\right)$. As a last example let $s \in \mathbb{\text { then }} f(x(s u) y)=s f(x u y)$.

Proposition 3.1. (i) The integral (3.1) converges absolutely, uniformly over compact subsets of

$$
\left\{(\lambda, x) \in t_{\Phi}^{*} \times G^{0} \mid \operatorname{Re}\left\langle\lambda+\rho-\rho_{c}, \alpha>>0 \text { for aach } \lambda \in \Delta_{c}^{+}\right\}\right.
$$

(ii) Furthermore any $u \in U\left(g_{\Phi}\right)$ can be applied to $\xi_{\lambda}^{0}$ by applying it to the integrand before the integration. Using the above definition this can be written

$$
\xi_{\lambda}^{\circ}\left(u^{\vee} x\right)=\int_{\bar{N}_{C}} e^{\left\langle-\lambda-\rho, H\left(x^{-1} u \bar{n}\right)\right\rangle} d \bar{n}, x \in G^{\circ} .
$$

(iii) Whenever well defined using (i) we have ${ }^{1)}$

$$
\xi_{\lambda}^{\circ}(e H)=c\left(-i\left(\lambda+\rho-\rho_{c}\right)\right)
$$

where $c(\cdot)$ is Harish-Chandras c-function corresponding to the Riemannian symmetric space $\mathrm{K}^{\circ} / \mathrm{K} \mathrm{H}_{\mathrm{H}}$.

Proof: For $\bar{n} \in \bar{N}_{c}$ we write, cf. Section 2,

$$
\text { (3.3) } \quad \bar{n}=k(\bar{n}) \exp (H(\bar{n})) n(\bar{n}) \in(K \cap H) T_{C}
$$

and find for $u \in U\left(g_{\downarrow}\right)$

$$
\begin{aligned}
& \int_{\bar{N}_{C}}\left|e^{\left\langle-\lambda-D, H\left(x^{-1} u \bar{n}\right)\right\rangle}\right| d \bar{n}= \\
& \int_{\bar{N}_{C}}\left|e^{\left\langle-\lambda-D, H\left(x^{-1} u k(\bar{n})\right)\right\rangle}\right| e^{R e\langle-\lambda-D, H(\bar{n})\rangle} d \bar{n} .
\end{aligned}
$$

1) The measure on $\bar{N}_{c}$ is normalized by

$$
\int \bar{N}_{c} e^{\langle-2 c} c^{\cdot H(\bar{n})\rangle} d \bar{n}=1 .
$$


Now $(\lambda, x, k) \rightarrow e^{\left.<-\lambda-\rho_{0} H\left(x^{-1} u k\right)\right\rangle}$ is a continuous function on $t^{*} \times G^{\circ} \times K H_{H} \cdot H a r i s h-$ Chandras c-function for $\mathrm{K}^{\circ} / \mathrm{k} \cap \mathrm{H}$ is given by, see [5],

$$
c\left(-i\left(\lambda+\rho-\rho_{c}\right)\right)=\int_{\bar{N}_{C}} e^{\left\langle-\left(\lambda+\rho-\rho_{c}\right)-\rho_{c}, H(\bar{n})\right\rangle} d \bar{n},
$$

with absolute and uniform convergence for $\lambda$ satisfying

$$
\operatorname{Re}\left\langle\lambda+\rho-\rho_{c}, \alpha\right\rangle>0 \text { for each } \lambda \in \Delta_{c}^{+}
$$

From this the proposition follows easily.

Theorem 3.2. Let $\lambda \in t^{\star}$ satisfy $(2.2),(2.3)$ and $(2.6)$, then

(i) $\quad \xi_{\lambda}^{\circ}$ is well defined, nonzero and $\mathrm{K}^{\circ}$-finite of irreducible $\mathrm{k}^{\circ}$-type $-\mu_{\lambda}$.

(ii) $\xi_{\lambda}=\eta^{-1}\left(\xi_{\lambda}^{0}\right)$ is the minimal spherical function for $T_{\lambda}$ corresponding to the dominant weight vector.

(iii) $\quad \int_{k \cap H} \xi_{\lambda}(k x) d k=c\left(-i\left(\lambda+\rho-\rho_{c}\right)\right) \psi_{\lambda}(x), \quad x \in \tilde{G}$.

Proof: Let $\lambda$ satisfy the conditions in Proposition 3.1 (i). Using the notation from the proof of that proposition we find

$$
\begin{aligned}
& \int_{K \cap H}^{-} \xi_{\lambda}^{\circ}(k x) d k=\int_{\bar{N}_{c}} \int_{K \cap H} e^{\left\langle-\lambda-\rho, H\left(x^{-1} k \bar{n}\right)\right\rangle} d k d \bar{n} \\
& =\int \bar{N}_{C} \int K H e^{\left\langle-\lambda-D, H\left(x^{-1} k k(\bar{n}) \exp (H(\bar{n})) n(\bar{n})\right)\right\rangle} d k d \bar{n} \\
& =\int_{\bar{N}_{C}} e^{\langle-\lambda-\rho, H(\bar{n})\rangle} d n \int_{K \cap H} e^{\left\langle-\lambda-\rho, H\left(x^{-1} k\right)\right\rangle} d k \\
& =c\left(-i\left(\lambda+o-\rho_{c}\right)\right) \psi_{\lambda}(x), \quad x \in G^{0} \text {. }
\end{aligned}
$$

This proves (iii) whenever $\xi_{\lambda}^{0}$ is $k^{0}$-finite, and also that $\xi_{\lambda}^{0} * 0$. In order to ensure using Proposition 3.1 (i), the convergence in (3.1) for $\lambda$ satisfying $(2.2),(2.3)$ and $(2.6)$ we need to know that 


\section{FLENSTED-JENSEN and K.OKAMOTO}

$$
\left\langle p-p_{c}, \alpha\right\rangle \geq 0 \text { for each } \alpha \in \Delta_{c}^{+}
$$

To prove this notice that the restriction of $\rho \sim_{-}^{-} \sim$ to $t$ equals $\rho-\rho c$, and that $2\left(\rho \sim-\rho_{c}\right)$ is a dominant weight of a finite dimensional subrepresentation of $k_{\downarrow}$ in $\Lambda^{1} p_{\downarrow}$, with $1=\operatorname{diq}\left(n \tilde{n} p_{\uparrow}\right)$. Therefore we have

$$
\left\langle\rho^{\sim}-\rho_{c}, B\right\rangle \geq 0 \text { for each } B \in \Delta_{c}^{\sim+} \text {. }
$$

Let now $\alpha \in \Delta_{c}^{+}$, then $\alpha$ is the restriction to $t$ of an element $B=\alpha+\alpha^{\prime} \in \Delta_{c}{ }^{+}$, where $\left\langle\alpha^{\prime}, t^{c}=0\right.$. Since $-\beta^{\top}=-$ Bot $=\alpha-\alpha^{\prime} \epsilon \Delta_{c}^{\sim+}$ we have

$$
\begin{aligned}
& \left\langle p-p_{c}, \alpha\right\rangle=\left\langle p \sim-p_{c}{ }_{c}^{, \alpha\rangle}\right. \\
& =\frac{1}{2}\left\langle D^{\sim}-D_{C}{ }_{C}, B-B^{\top}\right\rangle \geq 0,
\end{aligned}
$$

which is what we need. Thus $\xi_{\lambda}^{\circ}$ is well defined and non-zero.

To prove that $\xi_{\lambda}^{\circ}$ is $k^{\circ}$-finite, we $f i x$ any $x$ from $G^{\circ}$ and look at the function on $k^{\circ}$ :

$$
y \rightarrow e^{\left\langle-\lambda-\rho, H\left(x^{-1} y\right)\right\rangle}
$$

It belongs to the space $C_{\lambda_{1}}=C_{\lambda_{1}}\left(K^{0} / M_{t} \mathrm{TN}_{c}\right)$, where $\lambda_{1}=\lambda+\rho-\rho_{c}$, given by

$$
c_{\lambda_{1}}=\left\{\begin{array}{l|l}
\varphi \in c^{\infty}\left(k^{\circ}\right) & \begin{array}{l}
\varphi(y \operatorname{man})=e^{\langle-\lambda-\rho, H(a)\rangle} \varphi(y) \\
\text { for each } y \in x^{\circ}, m \in M_{t}, a \in T \\
\text { and } n \in N_{c}
\end{array}
\end{array}\right\} .
$$

The integration over $\overline{\mathbf{N}}_{C}$ is an intertwining operator taking $C_{\lambda_{1}}$ into $\bar{C}_{\lambda_{1}}=\bar{C}_{\lambda_{1}}\left(K^{\circ} / M_{t}{ }^{T \bar{N}_{c}}\right)$, which is defined like $C_{\lambda_{1}}$ but with the condition

$$
\varphi(y \operatorname{man})=e^{\left\langle-\lambda-\rho+2 \rho_{c} \cdot H(a)\right\rangle} \varphi(y) .
$$

Since $\mu_{\lambda}=\left(\lambda+p-2 p_{c}\right)$ satisfies $(2.6)$ the image of this intertwining operator is an irreducible finite dimensional representation of $k^{\circ}$ with dominant weight $-\mu_{\lambda}$ This finishes the proof of (i) and (iii). It follows now that $\xi_{\lambda}$ is well defined, that it is the dominant weight vector of the representation of $k^{0}$ generated by $\psi_{\lambda}$. Therefore (ii) is a consequence of Theorem 2.2 . 


\section{Discrete series}

Remark. Assume $\lambda$ as in Theorem 3.2. Then we know that $T_{\lambda}$ is irreducible, and it follows that any $\mathrm{K}^{2}$-finite vector for $T_{\lambda}$ is of the form $u \xi_{\lambda}$, for same $u \in U\left(g_{\downarrow}\right)$. By proposition 3.1 (ii) we have

$$
\left(u \xi_{\lambda}\right)^{\circ}(x)=\int_{\bar{N}_{c}} e^{\left\langle-\lambda-\rho, H\left(x^{-1} u \bar{n}\right)\right\rangle} d \bar{n}, x \in G^{\circ} .
$$

In particular if $u$ commutes with $\operatorname{Ad}\left(\overline{\mathbb{N}}_{c}\right)$ then

$$
\left(u \xi_{\lambda}\right)^{\circ}(x)=\int_{\bar{N}_{c}} e^{\left\langle-\lambda-\rho, H\left(x^{-1} \bar{n} u\right)\right\rangle} d \bar{n}, x \in G^{\circ} .
$$

This is the type of integral we want to study. So whenever there is absolute convergence, uniformly over a compact neighbourhood of $\lambda$ and over any compact subset of $G^{\circ}$ we define

$$
\xi_{\lambda, u}^{\circ}(x)=\int_{\bar{N}_{c}} e^{\left.\left\langle-\lambda-\rho, H(x)^{-1} \bar{n} u\right)\right\rangle} d \bar{n}, x \in G^{\circ} .
$$

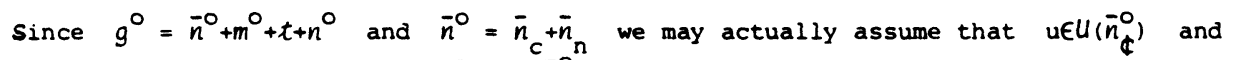
that $u$ is non-zero modulo $\left(\bar{n}_{c}\right) U\left(\bar{n}_{\Phi}^{\circ}\right)$. In other words we can express u using only elements from a basis of $\bar{n}_{n}$.

Let $S\left(\bar{n}_{n f^{\prime}}\right)$ be the complex symmetric algebra over $\bar{n}_{n}$. Let $\gamma: S\left(\bar{n}_{n \nsubseteq}\right) \rightarrow$

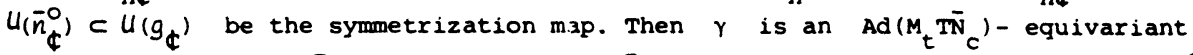
bijection between $S\left(\bar{n}_{n d}\right)$ and its image $\tau_{n}$, which is a cross section for $u\left(\bar{n}_{\dot{\phi}}\right)$ modulo $\left(\bar{n}_{c}\right) U\left(\bar{n}_{\mathbb{d}}\right)$.

Proposition 3.3. Let $u \in I_{n}$ and $u \neq 0$.

(i) There is a constant $c \geq 0$ such that $\xi_{\lambda, u}^{0}$ is defined provided ${ }^{\lambda} \in t_{\Phi}^{\star}$ satisfies

$$
\operatorname{Re}\langle\lambda, \alpha\rangle>C \text { for each } a \in \Delta_{c}^{+} \text {. }
$$

(ii) Whenever well defined by (i) we have that $\xi_{\lambda, u}^{0} \neq 0$, that $\xi_{\lambda, u}^{0} \in E_{\lambda}^{0}$ and that $\xi_{\lambda, u}^{0}$ is $\overline{\mathrm{N}}_{c}$-invariant.

(iii) If $u$ is $A d\left(\bar{N}_{c}\right)$-invariant, then we can take $c=0$.

Proof: (i). Let $\mathrm{V}$ be the finite dimensional, unipotent representation of $\overrightarrow{\mathrm{N}}_{c}$ generated by $u$ in $U_{n}$ using $\operatorname{Ad}\left(\bar{N}_{c}\right)$. 


\section{FLENSTED-JENSEN and $K$. OKAMOTO}

Choose a basis $u_{1}, \ldots, u_{s}$ for $v$, then

$$
\operatorname{Ad}(\bar{n}) u=\sum_{i=1}^{s} \varphi_{i}(\bar{n}) u_{i}, \bar{n} \in \bar{N}_{c} \text {. }
$$

where $\varphi_{i}, i=1, \ldots, s$ are polynomial functions on $\bar{N}_{c}$.

Notice that the function $y \rightarrow e^{\left\langle-\lambda-\rho, H\left(x^{-1} y\right)\right\rangle}$ for each fixed $x \in G^{0}$ belongs to $C_{\lambda}=C_{\lambda}\left(G^{\circ} / M^{0} \mathrm{TN}^{\circ}\right)$, where

$$
C_{\lambda}=\left\{\varphi \in C^{\infty}\left(G^{\circ}\right) \mid \begin{array}{c}
\varphi(y \operatorname{man})=e^{\langle-\lambda-\rho, H(a)\rangle} \varphi(y), \\
\text { for each } y \in G^{\circ}, m \in M^{\circ}, a \in T \text { and } n \in N^{\circ}
\end{array}\right\} .
$$

We now try to define a linear functional $T_{u}$ on $C_{\lambda}$ by

(3.6) $\left.\left\langle T_{u}, \varphi\right\rangle=\int_{\bar{N}_{C}} \varphi i \bar{n} u\right) d \bar{n}, \varphi E C_{\lambda}$.

We have using (3.3)

$$
\begin{aligned}
& \int_{\bar{N}_{C}}|\varphi(\bar{n} u)| d \bar{n} \leq \\
& \int_{\bar{N}_{C}}^{S} \sum_{i=1}^{S} \mid \varphi_{i}(\bar{n}) \varphi\left(u_{i} k(\bar{n}) \mid e^{R e<-\lambda-\rho, H(\bar{n})\rangle} d \bar{n} .\right.
\end{aligned}
$$

The proof of convergence of the integral defining the c-function, $c f$. the proof of Proposition 3.1, shows that the factor $e^{\operatorname{Re}\langle-\lambda-\mathcal{C}, H(\bar{n})\rangle}$ will dominate the $\varphi_{i}$ 's. when $\lambda$ satisfies the condition $\operatorname{Re}\langle\lambda, \alpha\rangle>c$ for each $a \in \Delta_{c}^{+}$for a suitably large $C$. This proves in particular that (i) holds.

By restricting functions in $C_{\lambda}$ to $H^{\circ}$ we get a bijection $\varphi \rightarrow \varphi^{\circ}$ between $C_{\lambda}$ and $C^{\infty}\left(H^{\circ} / M^{\circ}\right)$. The converse mapping is given by

$$
\varphi(x)=\varphi^{\prime}(k(x)) e^{\langle-\lambda-\rho, H(x)\rangle}, x \in G^{\circ} .
$$

From this and the estimates above it is clear, when the condition in (i) is satisfied, that $T_{u}$ defines a distribution $T_{\lambda, u}$ on $H^{\circ} / M^{\circ}=G^{\circ} / M^{\circ} T^{\circ}$ by

(3.7) $\left\langle T_{\lambda, u}, \varphi^{\prime}\right\rangle=\left\langle T_{u}, \varphi\right\rangle$. 


\section{Discrete series}

Notice that the support of $T_{\lambda, u}$ is contained in the $k^{0}$-orbit $0=0$ (1) in $\mathrm{G}^{\circ} / \mathrm{M}^{\circ} \mathrm{TN}^{\circ}$, where

$$
0=K^{\circ} M^{0} \mathrm{TN}^{\circ} / \mathrm{M}^{\circ} \mathrm{TN}^{\circ} \simeq \mathrm{K}^{\circ} / \mathrm{M}_{t} \mathrm{TN}_{\mathrm{C}} \simeq \mathrm{K} \cap_{\mathrm{H} / \mathrm{M}_{t}} .
$$

The $\lambda$-Poisson transform, $P_{\lambda}$, of a distribution $T$ on $H^{\circ} / M^{\circ}$ is defined by

$$
P_{\lambda}(T)(x)=\left\langle T, f_{\lambda, x^{\prime}}^{\prime}, \quad x \in G^{\circ}\right.
$$

where $f_{\lambda, x}^{\prime}$ is the restriction to $H^{\circ} / M^{\circ}$ of the function $f_{\lambda, x} \in C_{\lambda}$ given by

$$
f_{\lambda, x}(y)=e^{\left\langle-\lambda-\rho, H\left(x^{-1} y\right)\right\rangle}, \quad y \in G^{\circ} .
$$

It is now clear from $(3.4),(3.6),(3.7)$ and $(3.9)$ that $\xi_{\lambda}^{0}$ is equal to $P_{\lambda}\left(T_{\lambda}, u^{\prime}\right.$. Thus $\xi_{\lambda}^{0}$ belongs to $E_{\lambda}^{0}$. Since by our assumptions

$$
\operatorname{Re}\langle\lambda, \alpha\rangle>0 \text { for each } \alpha \in \Delta^{+} \text {, }
$$

it follows from Helgason [7], page 198, that $P_{\lambda}$ is injective. In order to finish the proof of $(i i)$ we just have to show that $T_{\lambda, u} \neq 0$. But this follows easily from (3.6), (3.7) and the facts that $\bar{N}^{\circ}=\bar{N}_{c} \exp \left(\bar{n}_{n}\right)$ and that the map $\overline{\mathrm{n}} \rightarrow \overline{\mathrm{n}} \mathrm{M}^{\circ} \mathrm{TN}^{\circ}$ of $\overline{\mathrm{N}}^{\circ}$ into $\mathrm{G}^{\circ} / \mathrm{M}^{\circ} \mathrm{TN}^{\circ}$ is injective. This proves (ii). Property (iii) follows directly from Proposition 3.1 .

We now turn to the question of $k^{\circ}$-finiteness of $\xi_{\lambda, u}^{0}$. From the remark before Proposition 3.3 it follows that if $\xi_{\lambda}^{0}$ is $k^{\circ}$-finite and $u \in U\left(\bar{n}^{\circ}\right)^{N_{c}}$ then $\xi_{\lambda, u}^{0}=u \xi_{\lambda}^{0}$, and $\xi_{\lambda}^{0}$ is thus $k^{0}$-finite.

If either $\xi_{\lambda}^{0}$ is not $k^{\circ}$-finite (for example if (2.6) is not satisfied) or if $u$ is not $\cdot \overline{\mathbf{N}}_{c}$-invariant, then we don't know whether or not $\xi_{\lambda, u}^{0}$ is $k^{0}$-finite. However we can derive some necessary conditions on $\lambda$ and $u$. We retain the notation from the proof of Proposition 3.3, and recall the definition (3.8) of $0_{\lambda}$ and that $\xi_{\lambda, u}^{0}$ is the Poisson transform of the distribution $T_{\lambda, u}$ which is supported on $0_{\lambda}$.

1) Recall that the Iwasawa decomposition depends on the choice of $\Delta^{+}=\Delta_{\operatorname{Re} \lambda}^{+}$ which again depends on $\lambda$. There are as many different orbits $0_{\lambda}$. as there are cosets in $w_{C} W$. These orbits are compact and minimal as $k^{\circ}$-orbits, cf. Matsuki [13]. 


\section{FLENSTED-JENSEN and R. OKAMƠTO}

Theorem 3.4. Let $\lambda \in t^{*}$ satisfy (2.2) and (2.3). Let $E_{\lambda}^{\infty 0}$ denote the set of $\mathrm{K}^{\circ}-$ finite functions in $E_{\lambda}^{\circ}$, which are Poisson transforms of distributions on $G^{\circ} / M^{\circ} \mathrm{TN}^{\circ}$ with support contained in $0_{\lambda}$. Then the following hold: (i) If $E_{\lambda}^{\infty} \neq\{0\}$ then

$$
\frac{\left\langle\mu_{\lambda}, \alpha\right\rangle}{\langle\alpha, \alpha\rangle} \in \mathbb{Z} \quad \text { for each } \quad \alpha \in \Delta_{c}^{+}
$$

where $\mu_{\lambda}=\lambda+p-2 p_{c}$

(ii) If $-\mu^{\sim}$ is the dominant weight of a $\tilde{K}^{\sim}$-type occurringin $E_{\lambda}^{\infty}$, then $\nu=\mu^{\sim}-\mu_{\lambda}$ is a linear combination of noncompact roots, i.e. roots from $\Delta\left(n_{n}, t\right)$ with nonnegative, integral coefficients.

Before we go tothe proof, we establish the following lemma.

Lemma 3.5. Let $G$ be a semisimple Lie group with Iwasawa decomposition $G=K A N$. Let $M$ be the centralizer of $A$ in $K$. Let $V$ be a finite dimensional representation of $G$. Let $g$ and $a$ be the Lie algebras of $G$ and $A$. Let $v \in V, v \neq 0$ satisfy the following, where $v \in a^{\star}$ :

$$
\begin{array}{lll}
\exp (H) \cdot v=e^{\langle V, H\rangle} v & \text { for each } & H \in a \\
m \cdot v=v & \text { for each } & m \in M
\end{array}
$$

then

$$
\frac{\langle\nu, \alpha\rangle}{\langle\alpha, \alpha\rangle} \in \mathbb{Z} \quad \text { for each } a \in \Delta(g, a)
$$

Proof: Let $\alpha \in \Delta(g, a)$. We can construct a subgroup $G^{\alpha}$ locally iusmorphic to $\operatorname{SL}(2, \mathbb{R})$ related to $\alpha$ such that $G^{\alpha}=K^{\alpha} A^{\alpha} N^{\alpha}$, where $K^{\alpha}=K \cap G^{\alpha}, A^{\alpha}=A \cap G^{\alpha}$ and $N^{\alpha}=N \cap G^{\alpha}$, and such that $M^{\alpha}=M G^{\alpha}$ is the centralizer of $A^{\alpha}$ in $K^{\alpha}$. cf. Helgason [6]page 75 .

Let $v^{\alpha}$ be the finite dimensional representation of $G^{\alpha}$ generated by $v . G^{\alpha}$ being isomorphic to $S L(2, \mathbb{R})$, it follows that $M^{\alpha}$ is equal to the center of $G^{\alpha}$. Since $v$ is $M^{\alpha}$-fixed, it follows that $M^{\alpha}$ acts trivially on $v^{\alpha}$. Let $g^{\alpha}$ and $a^{\alpha}$ be the lie algebras of $G^{\alpha}$ and $A^{\alpha}$, and let $<,>_{\alpha}$ denote the killing form on $g^{\alpha}$. A simple computation with $\operatorname{sL}(2, \mathbf{R})$ shows that, if $\bar{v}$ and $\bar{a}$ denote restriction to $a^{\alpha}$, then

$$
\frac{\langle\bar{v}, \bar{a}\rangle_{a}}{\langle\bar{a}, \bar{a}\rangle_{\alpha}} \in \mathbb{Z}
$$

but ([loc.cit.]page 75 (5)) 


$$
\frac{\langle v, \alpha\rangle}{\langle\alpha, \alpha\rangle}=\frac{\langle\bar{v}, \bar{\alpha}\rangle_{\alpha}}{\langle\bar{\alpha}, \bar{\alpha}\rangle_{\alpha}} \mathbb{z}
$$

Proof of Theorem 3.4: We start by proving (ii). So let $E_{-\mu} \sim$ be a $k^{0}$-irreducible subspace of $E_{\lambda}^{\infty}$ corresponding to the given $x^{0}$-type. Let $v_{-\mu} \sim$ be the set of distributions supported on $0_{\lambda}$ such that $E_{-\mu} \sim=P_{\lambda}\left(V_{-\mu} \sim\right)$. Let furthermore $f=P_{\lambda}(T)$ be a dominant weight vector for $E_{-\mu} \sim$. We are going to study $T$ in some details:

$\mathrm{T}$ is a linear functional on $C^{\infty}\left(G^{\circ} / M^{\circ} \mathrm{TN}{ }^{\circ}\right)$, which is, cf. (3.4), identified with $C_{\lambda}=C_{\lambda}\left(G^{\circ} / M^{\circ} T^{\circ}\right)$. As a linear functional on $C_{\lambda} T$ satisfies:

(i) $T$ is $\overline{\mathrm{N}}_{c}$-invariant, i.e. $\left\langle T, \varphi^{\bar{n}}\right\rangle=\langle T, \varphi\rangle$ for any $\varphi \in C_{\lambda}$ and any $\bar{n} \in \bar{N}_{c}$, where $\varphi^{\bar{n}}(\cdot)=\varphi\left(\bar{n}_{\bullet}\right)$.

$$
\begin{aligned}
& T \text { has } t^{\sim} \text {-weight }-\mu^{\sim} \text {, i.e. } \\
& \left\langle T, \varphi^{H}\right\rangle=\left\langle-\mu^{\sim}, H\right\rangle\langle T, \varphi\rangle \text { for any } \varphi \in C_{\lambda} \text { and any } H \in t^{\sim} \text {, where } \\
& \varphi^{H}(\cdot)=\varphi(H \cdot) \text {. }
\end{aligned}
$$

We now look at the restriction of $T$ to the open dense $\overline{\mathbf{N}}^{\circ}$-orbit $0_{1}$ of $\overline{\mathbf{N}}^{\circ}$ in $G^{\circ} / M^{\circ} \mathrm{TN}$. We parametrize $O_{1}$ by $\bar{N}^{\circ}$ via the bijective mapping

$$
\bar{n} \rightarrow \bar{n} M^{\circ} T^{\circ} \text {. }
$$

Notice that $\bar{N}_{c}$ parametrizes the open orbit $O_{0}$ of $\bar{N}_{c}$ in $0_{\lambda}$, or in other words $0_{0}=0_{1}$ no $\lambda$.

We now consider the distribution $T_{1}$ on $\bar{N}^{\circ}$ defined in the following way: Let $\varphi \in C^{\infty}\left(G^{\circ} / M^{\circ} \mathrm{TN}^{\circ}\right)$ and supp $(\varphi) \subset O_{1}$. Define $\varphi_{1} \in C_{C}^{\infty}\left(\bar{N}^{\circ}\right)$ by

$$
\varphi_{1}(\bar{n})=\varphi(k(\bar{n})) e^{\langle-\lambda-\rho, H(\bar{n})\rangle} .
$$

then let $\left\langle T_{1}, \varphi_{1}\right\rangle=\langle T, \varphi\rangle$. By the assumptions on $T$ we have that $T{ }_{1}$ is $\bar{N}_{c}-$ invariant and that $\operatorname{supp}\left(T_{1}\right) \subset \bar{N}_{c}$. But then $T_{1}$ must have the following form

$$
\left\langle T_{1}, \varphi_{1}\right\rangle=\int_{\bar{N}_{c}} \varphi_{1}(\bar{n} u) d \bar{n}, \quad \varphi_{1} \in C_{c}^{\infty}\left(\bar{N}^{-0}\right)
$$

where $u \in U\left(\bar{n}^{-}\right)$and $u$ can be expressed as a (noncommutative) polynomial in a basis for $\bar{n}_{n}$, (recall that $\bar{n}^{\circ}=\bar{n}_{c}+\bar{n}_{n}$ ). Notice that if $\varphi \in C_{\lambda}$ and $\operatorname{supp}(\varphi) \subset O_{1}$, then $\langle T, \varphi\rangle=\left\langle T_{1}, \varphi_{1}\right\rangle$ where $\varphi_{1}$ is the restriction of $\varphi$ to $\bar{N}^{0}$. We conclude that 
(3.10) $\langle T, \varphi\rangle=\int_{\bar{N}_{C}} \varphi(\bar{n} u) d \bar{n}=\left\langle T_{u}, \varphi\right\rangle$,

for each $\varphi \in C_{\lambda}$ with $\operatorname{supp}(\varphi) \subset O_{1}$. Notice that $T$ is an extension of the distribution $T_{u}$ defined by the integral formula $(3.10)$ on $0_{1}$. Also $T_{u}$ can at most have one $K^{0}$-finite extension with support contained in $0_{\lambda}$, since the difference between two extensions is again $k^{0}$-finite and has support contained in $0_{\lambda} 0_{0}$, but since the support must be a union of $k^{\circ}$-orbits it is empty by the minimality of $O_{\lambda}$.

Combining (3.10) with property (ii) of $T$ above we get for $\operatorname{supp}(\varphi) \subset 0_{1}$ and нet :

$$
\begin{aligned}
& \left\langle-\mu^{\sim}, H\right\rangle\left\langle T_{u^{\prime}}, \varphi\right\rangle=\int_{\bar{N}_{c}} \varphi(H \bar{n} u) d \bar{n} \\
& =\int_{\bar{N}_{c}}\left(\varphi(\bar{n}(a d H)(u))+\left\langle 2 \rho c^{\prime}, H>\varphi(\bar{n} u)+\varphi(\bar{n} U H)\right) d \bar{n}\right. \\
& =\left\langle T(\text { adH })(u)^{, \varphi}, \varphi+\left\langle 2 \rho c_{c}-\lambda-\rho, H\right\rangle\left\langle T_{u^{\prime}}, \varphi\right\rangle\right.
\end{aligned}
$$

or in other words

$$
\left\langle T{ }_{(a d H)(u)}, \varphi\right\rangle=\left\langle\mu_{\lambda}-\mu^{\sim}, H\right\rangle\left\langle T_{u}, \varphi\right\rangle .
$$

If we assume $u \in \bar{u}_{n}$, which we may, we conclude that

$$
\operatorname{ad}(H) u=\langle-v, H\rangle \text {, }
$$

where $v=\mu^{2}-\mu_{\lambda}$. This proves (ii).

To prove (1) first notice that $E_{\lambda}^{00}$ being nontrivial must contain a $x^{\circ}$-type with

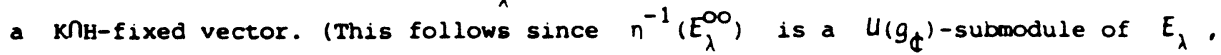
and as such it must contain a function, which is non-zero at the point eH). Returning again to the proof of (ii) we may assume that $E_{-\mu} \sim$ is such a $K^{0}-t y p e$. But then $\mu^{\sim} 1_{i t_{1}}=0$, such that $\mu \tilde{\epsilon} t^{*}$, also (3.11) $\frac{\left\langle\nu^{\sim}, a\right\rangle}{\langle\alpha, a\rangle} \in \mathbb{Z}^{+}$for each $a \in \Delta_{c}^{+}$.

Furthermore the dominant weight vector $T$, and thus $T_{u}$, is $M_{t}$-invariant .From this we conclude, cf. (3.10), that $u$ is $\operatorname{Ad}\left(M_{t}\right)$-invariant. Using Lemma 3.5 for 
$\mathrm{K}^{\circ}=\mathrm{K}_{\mathrm{H}} \mathrm{N}_{\mathrm{c}}$ we conclude that also

(3.12) $\frac{\langle-\nu, \alpha\rangle}{\langle\alpha, \alpha\rangle} \in \mathbb{Z}$ for each $a \in \Delta_{c}^{+}$.

combining (3.11) and (3.12) with $\mu_{\lambda}=\nu^{\sim}-v$, we have proved (i).

Corollary 3.6. Let $\lambda \in t^{\star}$ satisfy $(2.2),(2.3)$ and $(2.6)$. Then $T_{\lambda}$, the discrete series for $G^{\sim} / H$ generated by $\psi_{\lambda}$ or by $\xi_{\lambda}$, has a unique minimal $\kappa^{\sim}$-type with $\Delta_{c}^{-}$dominant weight $-\mu_{\lambda}$.

Proof: The proof is exactly as in Schlichtkrull [16], page 141, except that we can refer to our Theorem 3.4 instead of the reference to vogan-Speh [21]. In short: Let $-\mu \sim$ be a dominant weight of a $K^{\sim}$-type in $T_{\lambda}$, then

$$
\begin{aligned}
& \left\|\tilde{r}+2 \rho_{c} \sim^{2}=\right\| \mu_{\lambda}+2 \rho_{c} \tilde{c}^{2} \|^{2} \\
& =\left\|\mu_{\lambda}+2 \rho_{c}\right\|^{2}+\|v\|^{2}+2 \operatorname{Re}<\lambda+\rho, v \mid t^{+} \\
& +2 \operatorname{Re}<2 \rho_{m}, v_{\mid t_{1}}>\geq\left\|\mu_{\lambda}+2 \rho_{c}\right\|^{2} .
\end{aligned}
$$

and equality only holds for $v=0$.

From the proof of Theorem 3.4 we get the following

Corollary 3.7. Let $\lambda \in t^{*}$ and $u \in \bar{U}_{n}$ and assume that

$$
\xi_{\lambda, u}^{\circ}(x)=\int_{\bar{N}_{c}} e^{\left\langle-\lambda-\rho, H\left(x^{-1} \overline{n u}\right)\right\rangle} d \bar{n}, x \in G^{\circ},
$$

converges absolutely uniformly as in Proposition 3.3 (i). If $\xi_{\lambda, u}^{0}$ is $k^{\circ}$-finite giving rize to an irreducible representation of $k^{\circ}$ of dominant weight $-\mu \sim$, then (i) and (ii) of Theorem 3.4 are satisfied and $u$ satisfies

$$
\operatorname{Ad}(a) u=e^{\left\langle\mu_{\lambda}-\mu^{2}, H(a)\right\rangle} u \text {, for each } a \in T
$$

(ii) $\quad\left\{A d(m) u \mid m \in M_{t}\right\}$ generates an irreducible representation $\delta_{-\mu_{m}}$ of $M_{t}$ in $\bar{u}_{n}$ of $\Delta_{m}^{-}$-dominant weight $-\mu_{m}=-\mu_{1} \tau_{1}$. 


\section{FLENSTED-JENSEN and R. OKAMOTO}

Remarks. (a). The problem of the converse or Corollary 3.7 is the following: Assume that $u \in \bar{U}_{n}$ generates an irreducible representation $E_{-v}$ in $\bar{u}_{n}$ under $\operatorname{Ad}\left(M_{t} T\right)$ of weight $-v$. Let $v_{1}=v_{1 t}$ and $\mu_{m}=v_{1}$ such that $u$ is of $t$-weight $-v_{1}$ and generates an irreducible representation $\delta$ of $M_{t}$ with $\Delta_{m}^{-}$-dominant weight $-\mu_{\mathrm{m}}$. Assume furthermore that $\lambda \in t^{*}$ satisfies (2.2) and (2.3) and that $-\mu^{2}=-\left(\mu_{\lambda}+v\right)$ is a dominant weight for $k^{\circ}$.

The first question is whether $\xi_{\lambda, u}^{\circ}$ is at all defined, cf. (3.13) and Proposition 3.3? If so then notice that

(3.14) $\Phi_{x}: y+\int \bar{N}_{c} e^{\left\langle-\lambda-\rho, H\left(x^{-1} y \bar{n} u\right)\right\rangle} d \bar{n}$

for each $x \in G^{\circ}$ as a function on $K^{\circ}$ may be considered as belonging to the space of functions

$$
\left\{\begin{array}{l|l}
\varphi \in C^{\infty}\left(K^{0}, E_{-\nu}\right) & \begin{array}{l}
\varphi(y \operatorname{man})=\delta^{v}\left(m^{-1}\right) e^{\langle-\mu, H(a)\rangle} \varphi(y), \\
\text { for each } y \in K^{\circ}, m \in M_{t} \\
a \in T \text { and } \bar{n} \in \bar{N}_{c}
\end{array}
\end{array}\right\}
$$

This space, defining a non-unitary principal series for $k^{\circ}$, contains an irreducible finite dimensional subrepresentation with dominant weight $-\mu \sim$. The second question is, whether $\Phi_{x}$ belongs to this finite dimensional subspace for each $x \in G^{\circ}$ ?

(b). Assume that $\lambda \in t^{\star}$ satisfies $(2.2),(2.3),(2.7)$ and $(2.8)$ but not $(2.6)$. One might think that a minimal $k^{\circ}$-type in $E_{\lambda}^{\infty}$ could be constructed in the following way: choose $v$ as under $(a)$ in such a way that $\| \mu_{\lambda}+v+2 p_{c} \sim_{\|}$is minimal. Then prove that $\xi_{\lambda, u}^{0}$ is defined and $x^{\circ}$-finite.

(c). Theorem 3.4 is, at least for the linear case, contained in Theorem 3 of OshimaMatsuki [14], and our proof is much in the same spirit as the proof in [14].

(d). Corollary 3.7 clearly gives an upper bound on the multiplicity of any $\mathrm{K}^{2}-t y-$ pe $-\mu^{\sim}$ in any discrete series representations for $G^{\sim / H}$ contained in $n^{-1}\left(E_{\lambda}^{\infty}\right)$.

(e). Let $E_{\lambda}^{\infty}$ denote the set of $k^{\circ}$-finite functions in $E_{\lambda}^{\circ}$, which are Poisson transforms of hyperfunctions supported on $j_{\lambda}$. It follows from [14] that

$$
n^{-1}\left(E_{\lambda}^{\infty}\right) \subset n^{-1}\left(E_{\lambda}^{\infty}\right) \subset L_{\lambda}^{2}(G / H)
$$




\section{Discrete series}

As stated in [14] the square integrability holds for those $\lambda^{\text {'s }}$ for which the functions are $\mathrm{K}$-finite and not just $\mathrm{K}$-finite. But the asymptotic estimates needed does not seem to depend on the linearity of $G$ at all.

(f). From Oshima-Sekiguchi [15] and Oshima-Matsuki[14] it may very well follow, that for any $\tilde{K}$-finite and square integrabel function $f$ in $\tilde{E_{\lambda}}$, the asymptotic behaviour of $f^{\circ}=n(f)$ on $G^{\circ} / H^{\circ}$ is such, that $f^{\circ}$ is the Poisson transform of a distribution. If this is the case then $E_{\lambda}^{\infty 0}=E_{\lambda}^{\infty}$.

(g). It is also proved in [14] that for any $\lambda \in t^{\star}$, we have

$$
L_{\lambda}^{2}(G / H)^{K}=\underset{w}{\oplus} n^{-1}\left(E_{\lambda}^{\infty}\right)
$$

where $w$ runs over the elements in $w$, such that $w \cdot \lambda$ satisfies $(2.2),(2.3),(2.8)$ and a condition stronger than (2.7) which ensures that the occuring $K^{\sim}$-finite functions are actually $\mathrm{K}$-finite. 


\section{FLENSTED-JENSEN and $\mathrm{R}$. OKAYOTO}

\section{§ 4. The nonRiemannian isotropic spaces.}

In this section we turn to the semisimple symetric spaces of rank one. The Riemannian cases, i.e. the spaces of compact type (I) or the spaces of noncompact type (II), are from our point of view completely solved even for general rank. By this we mean that for case (I) $L^{2}$ (G/H) has a purly discrete spectrum, which is explicitly known. (See [4] Example 2.7 for an interpretation of this case in our framework). For case (II) Harish-Chandras spherical Plancherel formula shows that there are no discrete series. This also follows from Theorem 2.1 , since $K=H \neq G$ in this case and thus $\operatorname{rank}(\mathrm{G} / \mathrm{H})>\operatorname{rank}(\mathrm{K} / \mathrm{K} / \mathrm{H})=0$.

In Table 1 we give a list of the nonRiemannian semisimple symmetric spaces of rank one. We have also included some more information about these spaces. It follows in particular that Question $1^{\circ}$ of section 2 is relevant only for some of the isotropic spaces. For this reason we now turn to the isotropic spaces.

The isotropic spaces are well studied, see for example Strichartz [18] for the $\mathbf{R}$-hyperbolic spaces, Faraut [2] for the $\mathbf{R}-, \Phi$ and $\mathbf{H}$-hyperbolic spaces and Kosters [11] for the exceptional case. The only cases wich are not covered by these references are the simply connected covering spaces $G^{\sim} / H$ for the $\mathbf{R}$-hyperbolic spaces with $q=1$.

In Table 2 we describe for the isotropic spaces $G / H$ the values of $\lambda \in t^{\star}$ for which our discussion in Section 3 allow for the possibilityof having a discrete series for $\mathrm{G} / \mathrm{H}$. These parametervalues are easily related to the parametrization of the discrete series in the above references by comparring eigenvalues of the Casimir operator. This is also contained in Table 2. It follows in particular that there exists exactly one discrete series representation for each possible value of $\lambda$. Looking more carefully at [18], [2] and [11] one can also sort out all the $K$-types occurring in each $T_{\lambda}$. This means that as soon as we have answered Question $1^{\circ}$ of section 2 , we have also answered Question $2^{\circ}$. Strictly speaking this is only true when we consider the spaces $G / H$. For the $R$-hyperbolic spaces with $q=1$ we see no serious difficulties in generalizing either strichartz methods or Faraut's methods to give also the discrete series for $G^{\prime}, H$. But since we have not done this in any detail, we cannot claim to have answered Question $2^{\circ}$ for these simply connected covering spaces. 


\section{Discrete series}

We now turn to our discussion in Section 3. Elaborating on the results from section 3 we want to answer question $1^{\circ}$ of Section 2 , and at the same time we want to con-

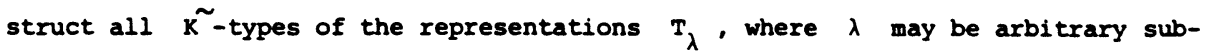
ject to conditions $(2.2),(2.3)$ and $(2.7)$, cf. Table 2 . In order to make our construction as simple and direct as possible, we want to do this without relying on the very explicit computations in [18], [2] and [11] or on the very deep results in Oshima-Matsuki [14].

Before coming to our main theorem we need some remarks and some lemmaes. Recall that $M_{t}$ and $M_{b}$ are the centralizers respectively of $t$ in $x N_{H}$ and of $b$ in $\mathrm{K} \cap \mathrm{H}$, where $b$ is maximal Abelian in $p \cap q$. If $B=\exp (b)$ then we have, $c f$. [3], that

$$
G=\mathrm{KBH}, \quad \mathrm{G}^{\sim}=\tilde{K}_{\mathrm{BH}} \text { and } G^{\circ}=\mathrm{K}^{\circ} \mathrm{BH} .
$$

Lemma 4.1. Every $\mathrm{K}^{\sim}$-type occurring in $C_{\mathrm{K}^{\infty}}^{\infty}\left(\mathrm{G}^{\sim} \cdot \mathrm{H}\right)$ has a nontrivial $\mathrm{M}_{\mathrm{b}}$-fixed vector.

Proof: Let $f \in C^{\infty}\left(G^{\sim} / H\right)$ and let $x \in G^{\sim}$ be such that $f(x) \neq 0$. According to (4.1) write $x=k b h$, let $k_{f}(g)=f(k g)$ and define $f_{1} \in c^{\infty}\left(G^{\sim} / H\right)$ by

$$
f_{1}(g)=\int_{M_{b}} k_{f}(m g) d m .
$$

Then $f_{1}$ is $M_{b}$-invariant and belongs to the $\mathrm{K}^{\sim}$-invariant subspace of $C^{\infty}\left(G^{\sim} / H\right)$ generated by $f \cdot f_{1}$ is nonzero since

$$
f_{1}(b)=\int M_{b} f(k m b) d m=f(k b) \neq 0 .
$$

This proves that any nontrivial $\mathrm{K}^{\sim}$-invariant subspace of $\mathrm{C}^{\infty}(\mathrm{G} / \mathrm{H})$ contains a nonzero $M_{b}$-invariant function, and the lemma follows.

Lemma 4.2. Let $\mathrm{G} / \mathrm{H}$ be nonRiemannian and isotropic, then

$$
\begin{aligned}
& k \cap H=M_{b} M_{t}, \\
& \text { i.e. every } k \in K \cap H \text { can be written } k=m_{1} m_{2} \text {, where } M_{1} \in M_{b} \text { and } m_{2} \in M_{t} \text {. }
\end{aligned}
$$




\section{FLENSTED-JENSEN and $\mathrm{K}$. OKAMOTO}

Proof: For the $\mathbf{R}-, \mathbf{F}$ - and $\mathbf{H}$-hyperbolic spaces this lemma follows easily from Table 3. For the exceptional space $F_{4(-20)} / \operatorname{Spin}(1,8)$ one can for example use the description of $F_{4(-20)}$ in Takahashi [19] and with a few computations see that $M_{b}$ and $M_{t}$ are both isomorphic to $\operatorname{spin}(7)$, but with two different embeddings. It follows then from Lemma 1 , page 534 of [19], that $M_{b} \simeq \operatorname{Spin}(7)$ acts transitively on $\mathrm{KH} / M_{t} \propto \operatorname{Spin}(8) / \operatorname{Spin}(7) \propto s^{7}$. This is the same as saying that $M_{b} M_{t}=K K_{H}$. 0

Lemma 4.3. Let $G / H$ be nonRiemannian and isotropic, then $\bar{n}_{c}$ and $\bar{n}_{n}$ commute with each other. In particular every element $u$ from $\bar{U}_{n}$ is $\operatorname{Ad}\left(\bar{N}_{c}\right)$-invariant, and $\xi_{\lambda, u}^{\circ}$ is well defined for all $\lambda$ such that $\left.\langle\lambda, \alpha\rangle\right\rangle 0$.

Proof. The possible roots in $\Delta^{+}$are $\alpha$ and $2 \alpha$. Therefore $\left[\bar{n}^{\circ}, \bar{n}^{\circ}\right] \subset g_{-2 \alpha}^{\circ}$, but from Table 1 it follows that $g_{-2 \alpha}^{0} \subset k^{0}$. From this we conclude that

$$
\left[\bar{n}_{c}, \bar{n}_{n}\right] \subset k^{\circ} n\left[k^{\circ}, p^{\circ}\right] \subset k^{\circ} n_{p}^{\circ}=\{0\}
$$

The rest of the lemma now follows from Proposition 3.3 .

Lemma 4.4. Let $G / B$ be nonRiemannian and isotropic. Every $A d\left(M_{t}\right)$-invariant nontrivial subspace of $U_{n}$ contains a nonzero $A d\left(M_{t} n M_{b}\right)$-fixed element.

Proof: Let $\lambda \in t^{*}$ be an arbitrary element satisfying $\langle\lambda, \alpha\rangle>0$ for each $\alpha \in \Delta^{+}$. Let $u \in U_{n}, u \neq 0$. We want to prove that the subspace generated by $u$ contains a nonzero $M_{t} M_{b}$-fixed element. Without loss of generality we may assume that $u$ is homogeneous under $T, i . e . ~ A d(a) u=e^{\langle-\psi H(a)\rangle} u$ for each $a \in T$ and some $v \in t^{*}$. It follows from Lemma 4.3 and Proposition 3.3 that

$$
\xi_{\lambda, u}^{\circ}(x)=\int_{\bar{N}_{c}} e^{\left\langle-\lambda-\rho H\left(x^{-1} n u\right)\right\rangle} d \bar{n}, x \in G^{\circ} .
$$

is well defined and nonzero. Choose $x \in G^{\circ}$ such that $\xi_{\lambda, u}^{0}(x) \neq 0$. Using (4.1) we can write $x=y^{-1} b h$, where $y \in K^{\circ}$. Furthermore using Lemma 4.2 write $y=m_{1} m_{2} a \bar{n}$, where $m_{1} \in M_{b}, m_{2} \in M_{t}, a \in T$ and $\bar{n} \in \bar{N}_{c}$. It is then easily seen that we have for every $m \in M_{t} n_{b}$

$$
0 \notin \xi_{\lambda, u}^{0}(x)=e^{\left\langle-\lambda-\rho+2 \rho_{c}-v, H(a)\right\rangle} \xi_{\lambda, u_{m}}^{0} \text { (b) , }
$$

where $u_{m}=\operatorname{Ad}\left(m_{2}\right) u$. Now 


$$
v_{1}=\int M_{t} n u_{b} u_{m} d m
$$

is $M_{t} \cap M_{b}$-invariant, nonzero and contained in the subspace of $\bar{u}_{n}$ generated under $\operatorname{Ad}\left(M_{t}\right)$ by $u$.

Recall that a pair $(M, L)$, where $M$ is a locally compact group and $L$ a compact subgroup, is called a Gelfand pair if the convolution algebra of continuous L-bi-invariant functions is commutative, or equivalently if every irreducible, unitary representation of $M$ has at most a one dimensional subspace consisting of L-fixed vectors.

Lemma 4.5. Let $G / H$ be nonRiemannian and isotropic. The pair $\left(M_{t}, M_{t} \cap_{M_{b}}\right)$ is a Gelfand pair.

Proof: From Table 3 it follows, when disregarding some trivial factors, that $\left(M_{t}, M_{t} \cap M_{b}\right)$ for the $\mathbf{R}-, \mathbb{C}$ - and $\mathbf{H}$-hyperbolic spaces are respectively

$$
\begin{aligned}
& (S O(p), S O(p-1)),\left(S(U(p) \times U(1)), s\left(U(p-1) \times U(1)^{\star} \times U(1)^{\star}\right)\right) \\
& \text { and }\left(\operatorname{sp}(p) \times S p(1), S p(p-1) \times S p(1)^{\star} \times S p(1)^{\star}\right) \text {. }
\end{aligned}
$$

These pairs are all known to be Gelfand pairs, since they occur as " $\mathrm{K} / \mathrm{M}$ " for the following rank one Riemannian symmetric space:

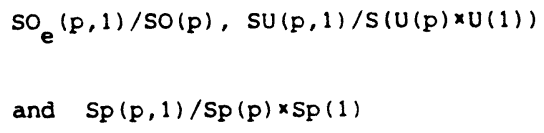

For the exceptional case we look at $\left(\operatorname{Spin}(7), G_{2}\right)$. As used before $\operatorname{Spin}(7) / G_{2} \propto S^{7}$. But SU(4) is contained in $\operatorname{Spin}(7)$ in such a way that already su(4) acts transitively on $s^{7}$ with stabalizer su(3). Therefore considering the $G_{2}$-bi-invariante continuous functions on $\operatorname{Spin}(7)$ as $G_{2}$-invariant functions on $S^{7}$, these form a subset of the $S U(3)$-invariant functions on $s^{7}$. Since $(S U(4), S U(3))$ is known to be a Gelfand pair it follows that so is $\left(\operatorname{Spin}(7), G_{2}\right)$. 
M. FLENSTED-JENSEN and $\mathrm{K}$. OKAMOTO

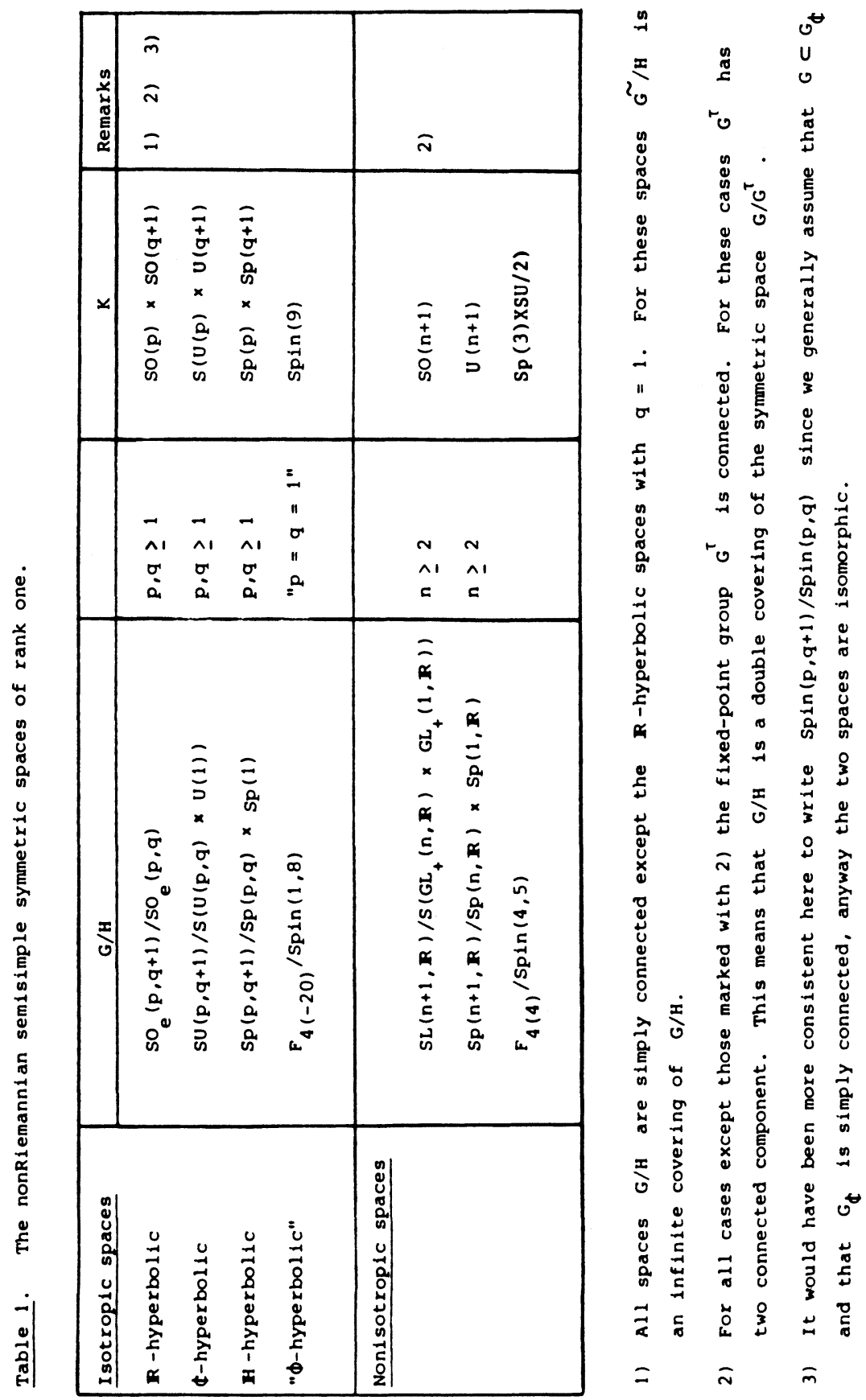


Discrete series

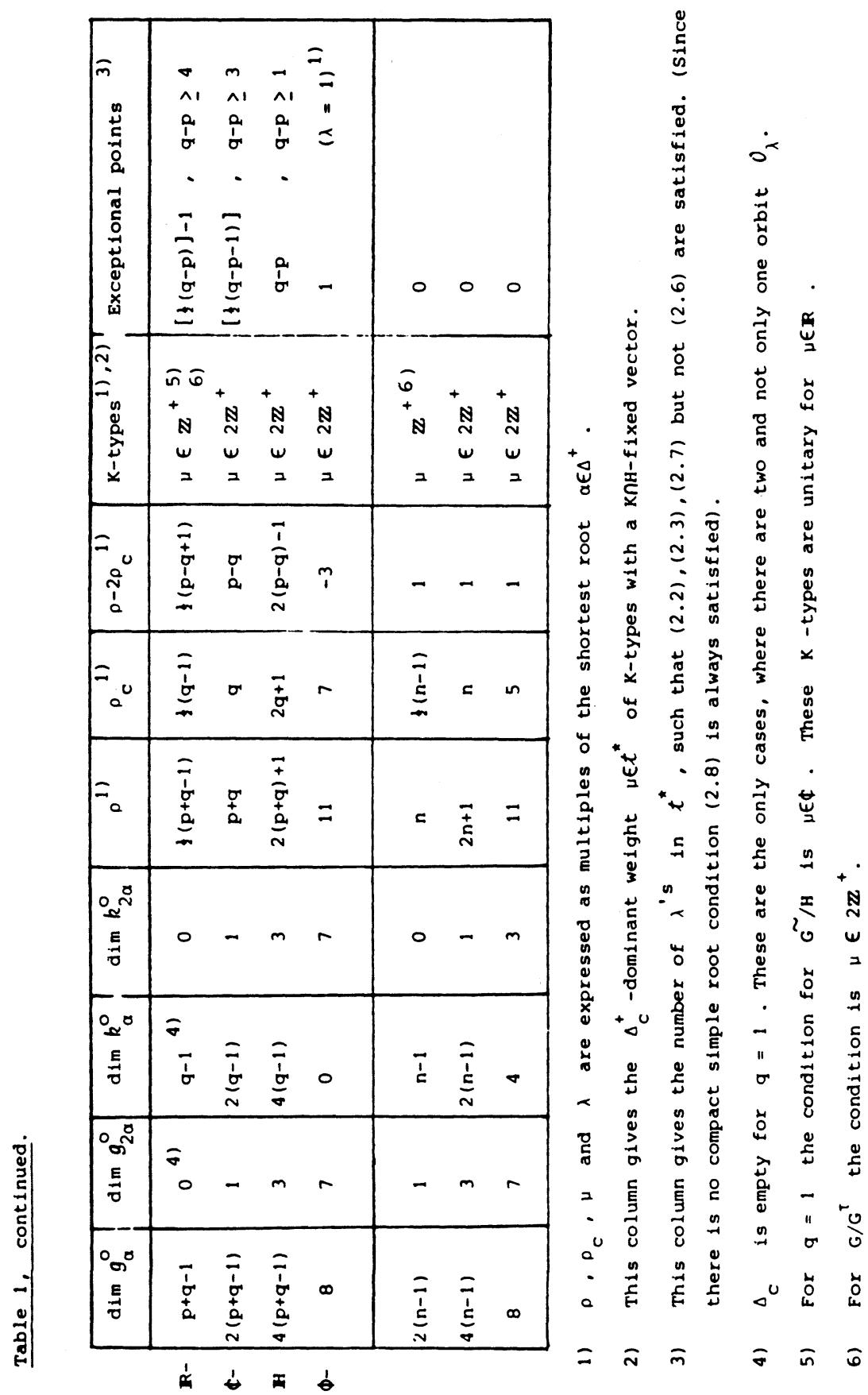


M. FLENSTED-JENSEN and K. OKAMOTO

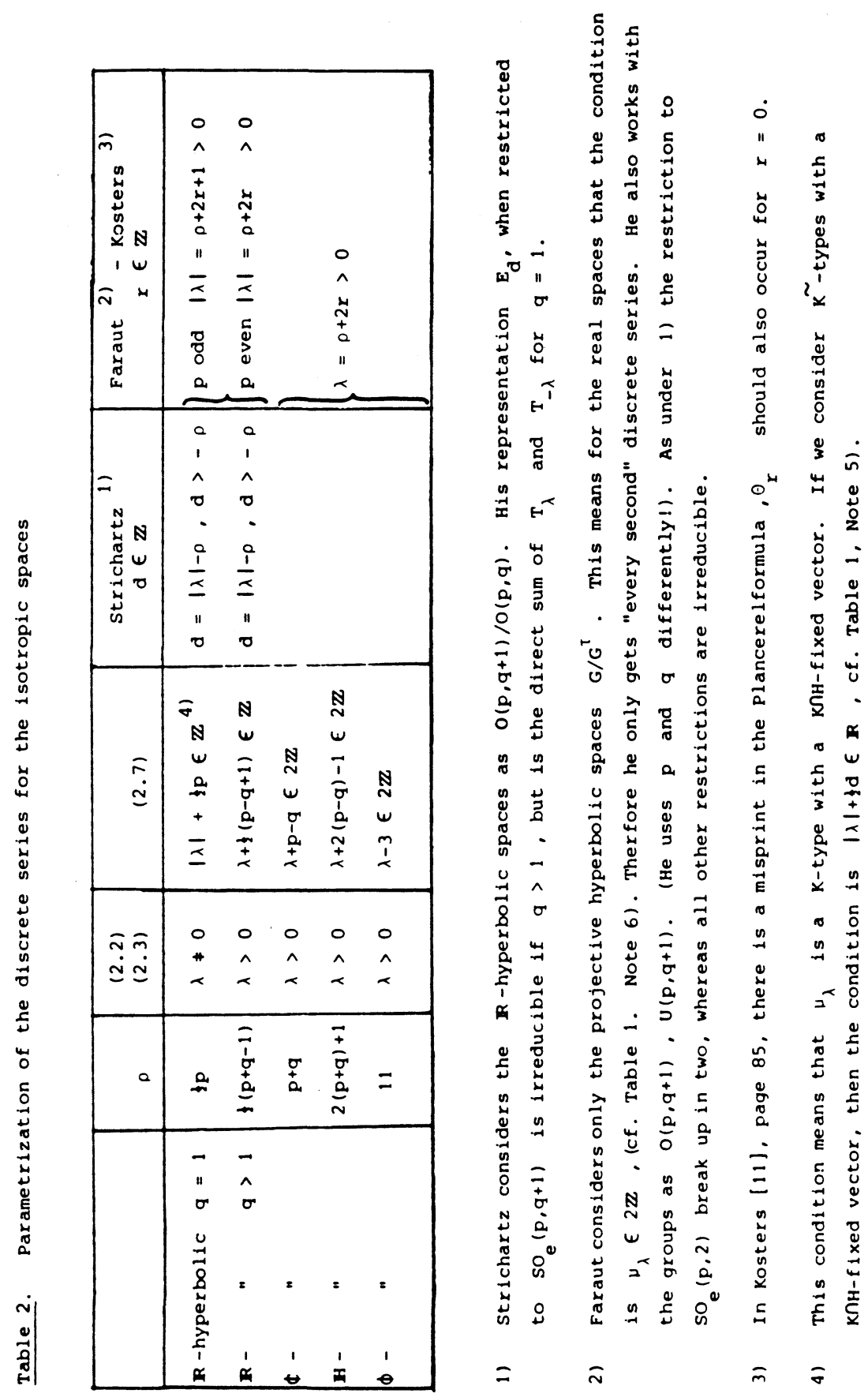




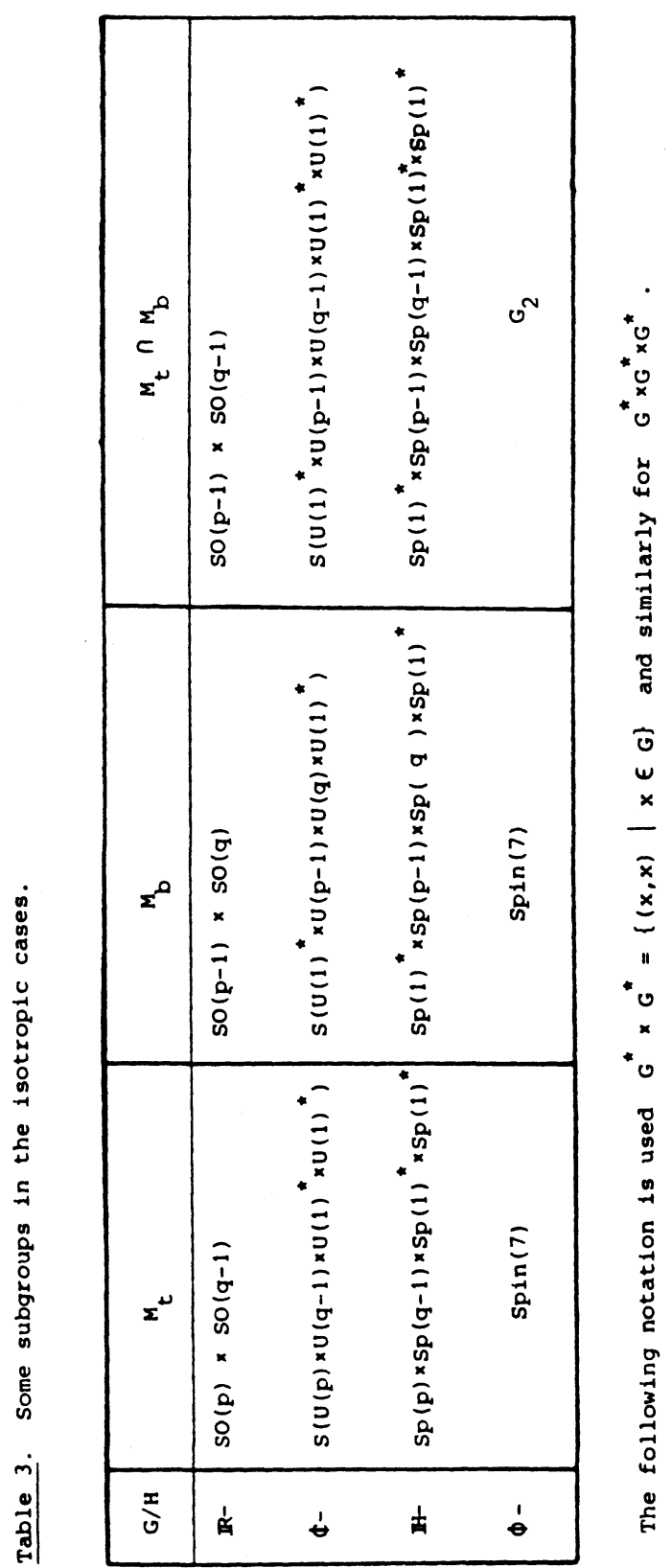




\section{PLENSTED-JENSEN and $\mathrm{K}$. OKAMOTO}

Definition. Let $\lambda \in t^{*}$ satisfy $\left.\langle\lambda, \alpha\rangle\right\rangle 0$ for each $\alpha \in \Delta^{+}$. Define $v_{\lambda}$ to be the set of weights $v \in\left(t_{\mathbb{f}}\right)^{*}$ which are $\Delta_{\mathrm{m}}^{+}$-dominant, and for which

$$
-v \text { is a weight for } t_{\downarrow} \text { in } u_{n} \text {. }
$$

(ii) $-\mu^{\tilde{L}}=-\left(\mu_{\lambda}+\nu\right)$ is a dominant weight for a finite dimensional representation of $\mathrm{K}^{\circ}$ having a nontrivial $\mathrm{M}_{\mathrm{b}}$-fixed vector.

Remark. (a). If $\lambda$ satisfies (2.2), (2.3) and (2.7), then $v_{\lambda}$ is nontrivial. To see this let $-v$ be the weight of any $M_{t}$-invariant element in $U_{n}$. Then by Lemma 3.5 we have that also $\mu^{\sim}=\mu_{\lambda}+\nu$ satisfies $(2.7)$. Now taking $\nu$ sufficiently large we can obtain that $\mu^{\sim}$ satisfies (2.6), which implies that $\nu \in V_{\lambda}$. (b). For $-\mu \sim$ to be of $\tilde{K}^{\sim}$-type contained in $E_{\lambda}^{00}$ it follows from Theorem 3.4 and Lemma 4.1 that it is necessary that $\mu^{\tilde{}-\mu_{\lambda} \in v_{\lambda}}$.

We are now ready to state the main theorem of this section. We state it in a rather general formulation in order to indicate what kind of more general results one might hope for. In Section 5 we shall do some more explicit computations of the functions involved.

Theorem 4.6. Let $\mathrm{G} / \mathrm{H}$ be a nonRiemannian, isotropic semisimple symmetric space. Let $\lambda \in t^{*}$ satisfy the conditions $(2.2),(2.3)$ and $(2.7)$. Let $\mu_{\lambda}=\lambda+p-2 \rho_{c}$.

(i) If $\left\langle\mu_{\lambda}, \alpha\right\rangle \geq 0$ for each $\lambda \in \Delta_{c}^{+}$then

$$
\xi_{\lambda}^{\circ}(x)=\int_{\bar{N}_{C}} e^{\left\langle-\lambda-D, H\left(x^{-1} \bar{n}\right)\right\rangle} d \bar{n}, x \in G^{\circ} .
$$

is well defined, $x^{\circ}$-finite and the dual function $\xi_{\lambda}$ is the dominant weight vector of weight $-\mu_{\lambda}$ of the unique minimal $K^{\sim}$-type of the discrete series representation $T_{\lambda}$ generated by $\xi_{\lambda}$. If $\left\langle u_{\lambda}, a\right\rangle\left\langle 0\right.$ for some $a \in \Delta_{c}^{+}$, then choose $v \in v_{\lambda}$ such that $\left\|\mu_{\lambda}+v+2 \rho_{c}^{\sim}\right\|=\operatorname{Min}\left\{\left\|\mu_{\lambda}+v^{0}+2 \rho_{c}\right\| \| v^{\cdot} \in v_{\lambda}\right\}$.

Let $u \in U_{n}$ be a $\Delta_{m}^{-}$-dominant weight vector corresponding to the weight $-v$, then 


\section{Discrete series}

(4.2) $\xi_{\lambda, u}^{\circ}(x)=\int_{\bar{N}_{c}} e^{\left\langle-\lambda-\rho, H\left(x^{-1} \bar{n} u\right)\right\rangle} d \bar{n}, \quad x \in G^{0}$,

is well defined, $\mathrm{K}^{\circ}$-finite and the dual function $\xi_{\lambda, u}$ is the dominant weight vector of weight $-\mu^{\sim}=-\left(\mu_{\lambda}+\nu\right)$ of a minimal $k^{\sim}$-type of the discrete series $T_{\lambda}$ generated by $\xi_{\lambda, u}$.

(iii) The $\boldsymbol{K}^{\sim}$-type decomposition of $T_{\lambda}$ is as follows: $-\mu \sim$ occurs in $T_{\lambda}$ if and only if $\mu^{\sim}-\mu_{\lambda} \in v_{\lambda}$.

(iv) If $-\mu^{\sim}$ occurs in $T_{\lambda}$, then the dominant weight vectors for this $\mathrm{K}^{\sim}$-type is given by $\xi_{\lambda, u}$, where $u$ is any $\Delta_{m}^{-}$-dominant weight vector for $M_{t} T$ in $a_{n}$ of weight $\mu_{\lambda}{ }^{-\mu}$.

Proof: Part (i) is just a special case of Theorem 3.2 and Corollary 3.6. For Part (ii) it follows from Lemma 4.3 that $\xi_{\lambda, u}^{0}$ is well defined. Taking into account Theorem 3.4 and its proof the two things left to prove are that (a) $\xi_{\lambda, u}^{\circ}$ is $K^{\circ}$-finite and that (b) $\xi_{\lambda, u}$ belongs to $L^{2}(G / H)$.

Proof of (a): Let $E$ be a Hilbert space for an irreducible finite dimensional representation $\pi$ of $\mathrm{K}^{0}$ with dominant weight $-\mu^{\sim}$. Chosen such that $\pi$ is unitary when holomorphically extended to $K^{\sim}$. E contains a unique subspace $F$ on which $M_{t} T$ acts as an irreducible representation $\delta$ of $\Delta_{m}^{-}$-dominant weight $-\mu \sim$. $F$ is isomorphic as a $M_{t} T$-module to the subspace of $\bar{u}_{n}$ generated by $u$ and therefore by Lemma 4.4 there is a nonzero $M_{t} M_{b}$-fixed vector $f_{0} \in F$, unique up to scalars. By assumption, $c f$. definition of $v_{\lambda}, E$ has a nontrivial $M_{b}$-fixed vector $e_{0}$. Now let $y \in K^{\circ}$ be written $y=m_{1} m_{2} a \bar{n}$, where $m_{1} \in M_{b}, m_{2} \leqslant M_{t}, a \in T$ and $\bar{n} \in \bar{N}_{c}$. We have

$$
\begin{aligned}
& \left(e_{0}, \pi(y) f_{0}\right)=\left(e_{0}, \pi\left(m_{1} m_{2} a \bar{n}\right) f_{0}\right) \\
& =e^{\left\langle-\mu^{\sim}, H(a)\right\rangle}\left(e_{0}, \pi\left(m_{2}\right) f_{0}\right)=e^{\left\langle-\mu^{\sim}, H(a)\right\rangle}\left(e_{0}^{0}, \pi\left(m_{2}\right) f_{0}\right),
\end{aligned}
$$

where $e_{0}^{0}$ is the orthogonal projection of $e_{0}$ onto $F$. The matrix coefficient $y \rightarrow\left(e_{0}, \pi(y) f_{0}\right)$ is nontrivial, so we conclude that $e_{0}^{\prime}$ is nontrivial. Clearly $e_{0}^{1}$ is $M_{t} M_{b}$-invariant. So by the uniqueness of $f_{0}$ we have that $e_{0}^{\prime}$ is a scalar multiple of $f_{0}$. Without loss of generality we can assume that $e_{0}^{1}=f_{0}$ and that $\left(e_{0}, f_{0}\right)=\left\|f_{0}\right\|^{2}=1$, or in other words we have

$$
\left(e_{0}, \pi(y) f_{0}\right)=e^{\left\langle-\mu{ }^{\sim}, H(y)\right\rangle}\left(f_{0^{\prime}} \delta\left(m_{2}\right) f_{0}\right),
$$




\section{FLENSTED-JENSEN and $\mathrm{K}$. OKAMOTO}

for each $y \in K^{\circ}$, where $y=m_{1} m_{2} \exp (H(y)) \bar{n}$ as above. (Notice that (4.3) shows that $e_{0}$ is uniquely determined, such that for $E$ the $M_{b}$-fixed vector is unique up to a scalar).

Let $u_{0}$ be the element in $U_{n}$ corresponding to $f_{0}$. From Lemma 4.4 and its proof it follows that it is enough to show that $\xi_{\lambda, u_{0}}^{0}$ is $k^{0}$-finite. It also follows
that

$$
\int_{M_{t} \cap M_{b}} \operatorname{Ad}\left(m m_{2}\right) u_{o} d m=\left(f_{o}, \delta\left(m_{2}\right) f_{o}\right) u_{0} .
$$

Let now $y \in x^{\circ}$ and $b \in B$ then we have (using same argument as in the proof of Lemma 4.4) that

$$
\xi_{\lambda, u_{0}}^{0}\left(y^{-1} b\right)=e^{\left\langle-\mu \mu^{\sim}, H(y)\right\rangle}\left(f_{0}, \delta\left(m_{2}\right) f_{0}\right) \xi_{\lambda, u_{0}}^{0}(b)
$$

where as before $y=m_{1} m_{2} \exp (H(y)) \bar{n}$. Combining (4.5) and (4.3) we get

$$
\xi_{\lambda, u_{0}}^{0}\left(y^{-1} b\right)=\left(e_{0}, \pi(y) f_{0}\right) \xi_{\lambda, u_{0}}^{0}(b)
$$

for every $y \in K^{\circ}$ and $b \in B$. This finishes the proof of (ii) Part (a) since by (4.1) every $x \in G^{\circ} / H^{\circ}$ can be written $x=y^{-1} b^{\circ}$, with $y \in K^{\circ}$ and $b \in B$.

Proof of (b): This follows from Oshima-Matsuki [14], cf. Remark (e) at the end of our section 3. Another and more direct proof of Part (b) is obtained by simple inspection of the asymptotic behaviour of $b \rightarrow \xi_{\lambda, u}^{0}$ (b) from our explicit formulas in section 5 . This finishes the proof of Part (ii).

For the proof of Part (iii) and (iv) notice that our proof of (ii) did not use the minimality of $v$ in the proof of the $x^{0}$-finiteness of $\xi_{\lambda, u}^{0}$. This means that $\xi_{\lambda, u}^{0}$ is $k^{0}$-finite if and only if $\mu^{\sim}-\mu_{\lambda} \in V_{\lambda}$. So what is left to prove is that $T_{\lambda}$ contains all the functions $\xi_{\lambda, u}$ constructed in this way. Using Theorem 3.4 this is equivalente to saying that $E_{\lambda}^{\infty}$ is an irreducible $u\left(g_{\uparrow}\right)$-module.

As noted before every $U\left(g_{\mathbb{t}}\right)$-submodule of $C_{K^{\circ}}^{\infty}\left(G^{\circ} / H^{\circ}\right)$ contains $K^{\circ}-t /$ hes with a nontrivial $\mathrm{K} \cap H-f i x e d$ vector. From the next Lemma 4.7 it follows that any $u\left(g_{\mathbb{\Phi}}\right)-$ submodule of $E_{\lambda}^{\infty}$ contains every $k \cap H$-fixed vector of every $K^{\circ}$-type occurring. Therefore $E_{\lambda}^{0{ }^{\lambda}}$ is irreducible. 
Lemma 4.7. Let $G / H$ be nonRiemannian and isotropic. Let $\bar{n}_{n}$ be equipped with an $M_{t}$-invariant inner product and let $x_{1} \ldots x_{s}$ be an orthonormal basis of $\bar{n}_{n}$. Define $\omega_{0}$ in $\tau_{n}$ by

$$
\omega_{0}=x_{1} \text { if } s=1
$$

and

$$
\omega_{0}=x_{1}^{2}+\ldots+x_{s}^{2} \text {, if } s>1 \text {. }
$$

Then $\omega_{0}$ is $M_{t}$-invariant, and every $M_{t}$-invariant element in $U_{n}$ is a polynomial in $w_{0}$.

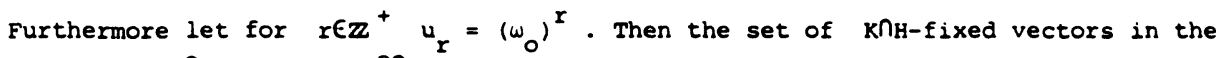
different $k^{\circ}$-types in $E_{\lambda}^{00}$ is up to scalars given by

$$
\left\{\xi_{\lambda, u_{r}}^{0} \mid r \in \mathbb{Z}^{+} \text {and } \mu_{\lambda}+c r \alpha \geq 0\right\}
$$

where $c=1$ if $s=1$ and $c=2$ if $s>1$. For any $r \in z$ we have

$$
\omega_{0} \xi_{\lambda, u}^{0}=\xi_{\lambda, u_{r+1}}^{0} .
$$

( $\alpha$ is the shortest root in $\Delta^{+}$).

Proof: Since $b$ is one dimensional we can assume that $b=\operatorname{Ir}\left(X_{1}-\tau\left(X_{1}\right)\right)$. From this we easily conclude that the stabilizer of $x_{1}$ in $M_{t}$ is $M_{t} M_{b}$. From Table 3 , cf. also the proof of Lemma 4.5, it follows that $M_{t} / M_{t} n_{b}$ is a sphere.

Now a checking of dimensions shows that $A d\left(M_{t}\right)\left(x_{1}\right)$ must be the connected component of the unit sphere in $\bar{n}_{n}$. From this follows that every $M_{t}$-invariant element in $\bar{n}_{n}$ is a polynomial in $\omega_{0}$.

Notice that the weight of $\omega_{0}$ is $-c \alpha$, where $c=1$ if $s=1$ and $c=2$ if $s>1$. From the proof of Theorem 3.4 (i) it follows, cf. (3.11) and (3.12), that the $\mathrm{K}^{2}$-type with weight $-\mu^{\sim}=-\left(\mu_{\lambda}+v\right)$ has a $\mathrm{k} n \mathrm{H}$-fixed vector if and only if the $u$ corresponding to $v$ is $M_{t}$-invariant and

$$
\frac{\left\langle v, \alpha_{1}\right\rangle}{\left\langle\alpha_{1}, a_{1}\right\rangle} \in \mathbb{E z} \text { for each } \alpha_{1} \in \Delta_{c}^{+} \text {. }
$$




\section{FLENSTED-JENSEN and $\mathrm{K}$. OKAMOTO}

Now this condition with $v=c a$ is always satisfied. (If $c=1$, then we are in the case of the $\mathbf{R}$-hyperbolic space with $p=1$, and $a_{1}=2 \alpha$ is not a root. In all other cases $c=2$, and the condition is satisfied even with $\alpha_{1}=2 \alpha$ ). This

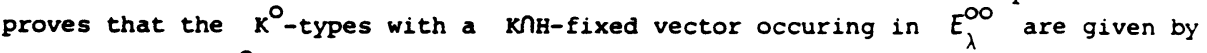
the functions $\xi_{\lambda, u_{\mu}}^{0}$, for which $\mu_{\lambda}+\operatorname{cr} \alpha \geq 0$. The last statement in the Lemma follows from Proposition 3.1 (ii) and the fact that $\omega_{0}$ is $\operatorname{Ad}\left(\overline{\mathrm{N}}_{c}\right)$-invariant, cf. Lemma 4.3 .

Remark. (a). We have already mentioned that Table 2 shows that it can be concluded from the work of Strichartz [18], Faraut [2] and Kosters [11], that the $T_{\lambda}{ }^{\prime}$ of Theorem 4.6 exhaust the discrete series for $G / H$. It follows from Table 1 that $G^{\sim} / H=G / H$ for all cases except for the $R$-hyperbolic spaces with $q=1$. The same conclusion for $G / H$ can be drawn from Oshima-Matsuki [14], cf. Remarks (e), (f) and $(g)$ at the end of section 3, provided one can show that $E_{\lambda}^{\infty}=E_{\lambda}^{\infty}$. But this last fact should be particularly easy to show in these cases, where $\operatorname{dim}(b)=1$.

(b). The minimal $\mathrm{k}$-types. If $\mu_{\lambda} \geq 0$ the minimal $\tilde{k}$-type is $-\mu_{\lambda}$. If $\mu_{\lambda}<0$ and $\mu_{\lambda}$ is even say $\mu_{\lambda}=-2 r, r \in N$, then by Lemma 4.7 we have that $0=\mu_{\lambda}+2 r \alpha$ is a $K$-type of $T_{\lambda}$. This means that the trivial $K$-type is contained in $T_{\lambda}$. clearly the trivial $\mathrm{K}$-type is minimal. So far all these minimal $\mathrm{K}^{\tilde{N}-t y p e s}$ have a $\mathbf{K} \cap \mathrm{H}$-fixed vector. Now assume that $\mu_{\lambda}=-2 r+1<0$ with $r \in \mathbf{N}$. This is only possible, cf. Table 1 and 2 , if $G / H$ is $R$-hyperbolic and if $q \geq p+4$. Now if also $p>1$, then Lemma 4.7 shows that the trivial $K$-type is not contained in $T_{\lambda}$. The minimal

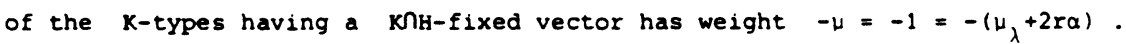

Exampie 4.8. Let $G / H=S O_{e}(2,7) / S O_{e}(2,6)$. Let $\lambda=t a$, then $u_{\lambda}=-\alpha$. Let $B E \Delta^{\sim}$ be chosen such that $B_{\mid t}=\alpha$ and $x_{-B} \in \bar{n}_{n}$, (there are two possible choices of $B$ ). It is easily seen that, with $u=x_{-B} \in D_{n}$, we have that $u$ is $M_{b} M_{t^{-}}$ fixed. Now $-\mu^{\sim}=-\left(\mu_{\lambda}+v\right)=\alpha-B$, which is the weight of the following one dimensional representation $\pi \pm 01$ of $K=S O(2) \times S O(7)$, where + or - is chosen according to the choice of $B$,

$$
\pi=\left\{\begin{array}{cc}
\cos \theta & -\sin \theta \\
\sin \theta & \cos \theta
\end{array}\right\}=e^{+10} .
$$

Since $y_{b}=S O(5)$ we have that $\pi \pm 01$ has a $M_{b}$-fixed vector. Thus $\pi^{ \pm} \cdot 1$ are $K$-types of $T_{\lambda}$. Finally a simple computation shows that 


\section{Discrete series}

$\left\|\mu^{\sim}+2 \rho_{c}\right\|^{2}=1+\left\|2 \rho_{m}\right\|^{2}+\left\|2 \rho_{c}\right\|^{2}$

and

$$
\left\|\mu+2 \rho_{c}\right\|^{2}=\left\|2 \rho_{m}\right\|^{2}+\left\|1+2 \rho_{c}\right\|^{2} \text {. }
$$

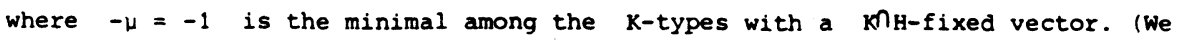
have normalized such that $\alpha=1$ ). From Table 1 we get that $\rho_{c}=5 / 2$ and thus

$$
\begin{aligned}
& \left\|\mu^{2}+2 \rho_{c}\right\|^{2}=26+\left\|2 \rho_{m}\right\|^{2}< \\
& <36+\left\|2 \rho_{m}\right\|^{2}=\left\|\mu+2 \rho_{c}\right\|^{2} .
\end{aligned}
$$

This means that $\pi^{+} \cdot 1$ and $\pi^{-} \cdot 1$ are two different minimal K-types, and neither of them have a $\mathrm{k}$ H $\mathrm{H}$-fixed vector.

Remark. (c). Continuing the Remark (b) above it is easy to generalize Eksample 4.8 to show that if $\mu_{\lambda}$ is an odd negative integer and $p>1$, then no minimal $K-t y-$ pe of $T_{\lambda}$ has a $k n_{H}$-fixed vector. Furthermore if $P=2$ then $T_{\lambda}$ has two different minimal $\mathrm{K}$-types. 


\section{FLENSTED-JENSEN and $\mathbf{K}$. ORAMOTO}

§5. Explicit formulas for the isotropic spaces.

The purpose of this section is to give an explicit evaluation of the integral (4.2) defining $\xi_{\lambda, u}^{0}$. We assume that $u$ is $M_{t} M_{b}-i n v a r i a n t$ and belongs to an $M_{t} T-i r-$ reducible subspace of $U_{n}$ with $\Delta_{m}^{-}$-dominant weight $v \in v_{\lambda}$. This means that $\xi_{\lambda, u}^{0}$ is $\mathrm{K}^{0}$-finite of irreducible type $-\mu^{\sim}=-\left(\mu_{\lambda}+\nu\right)$. By the help of $(4.6),(4.3)$ and (4.1) we have an explicit formula for $\xi_{\lambda, u}^{\circ}$ as soon as we know it on $B$. However on $B \xi_{\lambda, u}^{\circ}$ agrees with $\xi_{\lambda, u}$, cf. the Duality Theorem. Actually formula (4.6) is very convenient for the point of view of duality, since it shows that$$
\text { (5.1) } \quad \xi_{\lambda, u}\left(k^{-1} b\right)=\left(e_{0}, \pi(k) f_{0}\right) \xi_{\lambda, u}^{0}(b)
$$

for $k \in K^{\sim}$ and $b \in B$.

We shall not go into the explicit parametrization and description of the special functions on the groups $K^{\sim}$ and $M_{t}$, which are involved in evaluating $\left(e_{0}, \pi(k) f_{0}\right)$.

Let in the following $\mathbb{F}=\mathbb{R}, \$$, I or $\Phi$ respectively according to whether we treat the $\mathbb{R}, \mathbb{\Psi}$ - or $\mathbb{H}$-hyperbolic spaces or the exceptional space. Because of the non-associativity of the Cayley numbers $\varphi$, what we write in the following for $F=\varphi$ is not really correct. One should use the model for the exceptional space used in Takahashi [19] and Kosters [11]. However the formulas obtained, at least formula (5.7), also hold for this case.

We take our group $G^{0}$, which is respectively $s_{e}(p+q, 1), s u(p+q, 1), S p(p+q, 1)$ and $F_{4(-20)}$, to be an $(p+q+1) \times(p+q+1)$ matrix group over the field $\mathbb{F}$. The involution $T$ is given by conjugation with the matrix

$$
I_{p+q, 1}=\left\{\begin{array}{rrrr}
1 & 0 & \ldots & 0 \\
0 & & & . \\
\vdots & & & \vdots \\
0 & 1 & 0 \\
0 & 0 & -1
\end{array}\right) \text {. }
$$

and $\sigma$ is given by conjugation with the matrix

$$
I_{p, q+1}=\left\{\begin{array}{cc}
I_{p} & 0 \\
0 & -I_{q+1}
\end{array}\right\} .
$$




\section{Discrete series}

We shall need the following subgroups

$T: \quad\left\{\begin{array}{ccc}I_{p+q-1} & 0 & 0 \\ 0 & \text { chs } & \text { shs } \\ 0 & \text { shs } & \text { chs }\end{array}\right\}, \quad \mathbf{s} \in \mathbf{R}$

$B: \quad b_{t}=\left\{\begin{array}{ccc}c h t & 0 & \text { sht } \\ 0 & I_{p+q-1} & 0 \\ \text { sht } & 0 & c h t\end{array}\right\}, \quad t \in \mathbb{R}$

$\bar{N}^{0}: \bar{n}(x, y, z)=\left\{\begin{array}{cccc}I_{p} & 0 & x & x \\ 0 & I_{q-1} & y & y \\ -\bar{x}^{-t} & -\bar{y}^{-t} & 1-\gamma & -\gamma \\ \bar{x}^{-t} & \bar{y}^{-t} & \gamma & 1+\gamma\end{array}\right\}, \quad y \in F^{q-1}$

where

$$
\gamma=\gamma_{x, y, z}=\frac{1}{z}\left(|x|^{2}+|y|^{2}+2 z\right) \text {. }
$$

Notice that $\overline{\mathrm{N}}_{c}$ is the subgroup obtained with $x=0$, and that $\exp \left(\bar{r}_{n}\right)$ is obtained by taking $y=z=0$.

Let $v_{0}=(0, \ldots, 0,1) \in \mathbb{F}^{p+q+1}$ and let $v_{1}=(0, \ldots, 0,1,1) \in \mathbb{F}^{p+q+1}$. If we as in Section 4 identify $t_{\downarrow}^{*}$ with $₫$ in such a way that the shortest root $a$ in $\Delta^{+}$is identified with 1 we get for any element $g \in G^{\circ}$ and any $\lambda \in t_{\downarrow}^{*}$ that

$$
e^{\langle-\lambda-D, H(g)\rangle}=\left|\left\langle v_{0}, g v_{1}\right\rangle\right|^{-\lambda-\rho} \text {. }
$$

Let now $g \in G^{\circ}$ be arbitrary. We write $g^{-1}$ in the following form

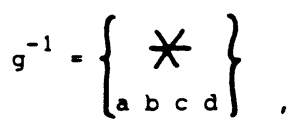

where $a \in F^{p}, b \in F^{q-1}, c, d \in F$ and furthermore

$$
|a|^{2}+|b|^{2}+|c|^{2}-|a|^{2}=-1 .
$$

We now take 


$$
\Phi=\Phi(x, y, z, a, b, c, d)=\left\langle v_{0}, g^{-1} \bar{n}(x, y, z) v_{1}\right\rangle,
$$

such that $e^{\left\langle-\lambda-\rho, H\left(g^{-1} \bar{n}\right)\right\rangle}=|\Phi|^{-\lambda-\rho}$.

A straight forward computation shows that

$$
\Phi=2 a \cdot x+2 b \cdot y+(d+c)+(d-c)\left(|x|^{2}+|y|^{2}+2 z\right)
$$

A few tedious computations show that

$$
\begin{aligned}
\Phi & =(d-c)\left(|x+\bar{a} \cdot|^{2}+|y+\bar{b} \cdot|^{2}+|d-c|^{-2}+\right. \\
& \left.+2 \operatorname{Im}\left(a \cdot \cdot x+b \cdot \cdot y+|d-c|^{-2} \bar{d} c\right)+2 z\right) .
\end{aligned}
$$

where $a^{\prime}=(d-c)^{-1} a$ and $b^{\prime}=(d-c)^{-1} b$, and therefore

(5.3) $|\Phi|^{2}=|d-c|^{2}\left[\left(|x+\bar{a} \cdot|^{2}+|y+\bar{b} \cdot|^{2}+|d-c|^{-2}\right)^{2}+|z \cdot|^{2}\right]$,

where

$$
z^{\prime}=2\left(z+\operatorname{Im}\left(a^{\prime} \cdot x+b^{\prime} \cdot y+|d-c|^{-2} \bar{d} c\right)\right)
$$

We now compute for fixed $g \in G^{\circ}$ and $x \in \boldsymbol{F}^{p}$

$$
I(\lambda, g, x)=\int_{\bar{N}_{C}} e^{\left\langle-\lambda-\rho, H\left(g^{-1 \bar{n} \bar{n}(x, 0,0))\rangle} d \bar{n} .\right.\right.}
$$

As the measure on $\overline{\mathbf{N}}_{c}$ we take dyd(2z) (which is normalized differently from $d \bar{n}$ in Proposition 3.1). Notice that $d z^{\prime}=d(2 z)$ and that $d y=d\left(y+\bar{b}^{\prime}\right)$. Let

$$
\omega^{2}=\left(\left|x+\bar{a}^{0}\right|^{2}+|d-c|^{-2}\right), \omega>0 .
$$

we find

$$
\begin{aligned}
& I(\lambda, g, x)=|d-c|^{-(\lambda+0)} \int_{F^{g-1}} \int_{\operatorname{Im}(\boldsymbol{F})}\left(\left(\omega^{2}+y^{2}\right)^{2}+z^{2}\right)^{-1(\lambda+0)} d z d y \\
& \left.\left.=r_{\lambda}|d-c|^{-(\lambda+p)}\right)_{\omega}^{\left(-2(\lambda+p)-2+(q+1) d i m_{R}\right.}(\mathbf{F})\right),
\end{aligned}
$$


where

$$
\begin{aligned}
r_{\lambda} & =\int_{F} q-1 \int \operatorname{Im}(F)\left(\left(1+|y|^{2}\right)^{2}+|z|^{2}\right)-\frac{1}{2}(\lambda+o) d z d y . \\
& =I(\lambda, e, 0)=\text { const } \cdot c(-i(\lambda+p-p)) .
\end{aligned}
$$

The last equality follows from Proposition 3.1 (iii). From Table 1 we find that $(q+1) \operatorname{dim}(\mathbb{R})-2=2 \rho_{c}$. Combining all this we have

$$
I(\lambda, g, x)=\gamma_{\lambda}|d-c|^{-(\lambda+\rho)} \omega^{-2\left(\lambda+\rho-\rho_{c}\right)}
$$

$(5.5)$

$$
=\gamma_{\lambda}|d-c|^{\mu \lambda}\left(1+|(\bar{d}-\bar{c}) x+\bar{a}|^{2}\right)-\left(\lambda+\rho-\rho_{c}\right)
$$

Let now $u \in U_{n}$ be expressed in terms of $x$ as $u=P\left(\frac{\partial}{\partial x}\right)$, then we get

$$
\xi_{\lambda, u}^{0}(g)=c\left(-i\left(\lambda+\rho-\rho, c^{\prime}\right)|d-c|^{\mu} \lambda_{p}\left(\frac{\partial}{\partial x}\right)\right.
$$

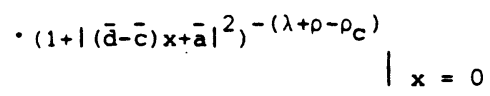

Theorem 5.1. Let $\mathrm{G} / \mathrm{H}$ be a nonRiemannian, isotropic semisimple symmetric space. Let $\lambda \in t_{t}^{*}$ satisfy $\left.\operatorname{Re}\langle\lambda, \alpha\rangle\right\rangle$, where $\alpha$ is the shortest root in $\Delta^{+}$. Let $u \in \bar{U}_{n}$ be homogeneous of degre $m$, and let $u$ expressed in the variables $x$ be $u=P\left(\frac{\partial}{\partial x}\right)$. We have

(5.7)

$$
\xi_{\lambda, u}^{0}\left(b_{t}\right)=c\left(-i\left(\lambda+o-o_{c}\right)\right)(\cosh t)^{\left(\omega_{\lambda}+m\right)} .
$$

$$
\left.\cdot P\left(\frac{\partial}{\partial x}\right)\left(1+|x|^{2}\right)^{-(\lambda+o-0} c^{\prime}\right|_{x=(-\sinh t, 0, \ldots, 0)}
$$

Proof: This follows from formula (5.6) taking $g=b_{t}$, which means that $a=(-\operatorname{sht}, 0, \ldots, 0), b=0, c=0$ and $d=c h t$. 


\section{FLENSTED-JENSEN and K. OKAYOTO}

\section{REFERENCES}

[1] Berger,M., Les espaces symétriques non compacts. Ann. Sci. École Norm. Sup., 74 (1957), 85-177.

[2] Faraut, J., Distributions sphériques sur les espaces hyperboliques. J. Math. pures et appl. 58 (1979), 369-444.

[3] Flensted-Jensen, M., Discrete series for semisimple symmetric spaces. Ann. of Math. 111 (1980), 253-311.

[4] Flensted-Jensen, M., Harmonic analysis on semisimple symmetric spaces. A method of duality. To appear in the proceedings of the Special Year in Lie Groups. University of Maryland, 1982-83. (Springer Lecture Notes in Mathematics).

[5] Harish-Chandra Spherical functions on a semisimple Lie group I and II. Amer. J. Math. 80 (1958), 241-310 and 553-613.

[6] Helgason, S., A duality for symmetric spaces with applications to group representations.

Adv. Math. 5 (1970), 1-154.

[7] Helgason, S., A duality for symmetric spaces with applications to group representations II. Differential equations and eigenspace representations. Adv. Math. 22 (1976), 187-219.

[8] Helgason, S., Differential geometry, Lie groups and symmetric spaces. Academic Press, New York-San Francisco-London 1978.

[9] Helgason, S., Groups and geometric analysis I. To appear Academic Press.

[10] Kashiwara, M., Kowata, A., Minemura, K., Okamoto, K., Oshima, T. and Tanaka, M., Eigenfunctions of invariant differential operators on a symmetric space.

Ann. of Math., 107 (1978), 1-39.

[11] Kosters,M.T., Spherical distributions on rank one symmetric spaces. Thesis, University of Leiden, 1983.

[12] Loos, O., Symmetric spaces. I, II. New York-Amsterdam, W.A. Benjamin, Inc., 1969. 


\section{Discrete series}

[13] Matsuki, T., The orbits of affine symmetric spaces under the action of minimal parabolic subgroups, J. Math. Soc. Japan 31 (1979), 331-357.

[14] Oshima, T. and Matsuki, T., A description of discrete series for semisimple symmetric spaces.Preprint 1983.

[15] Oshima, T. and Sekiguchi, J.: Eigenspaces of invariant differential operators on an affine symmetric space, Inventiones Math. 57 (1980), 1-81.

[16] Schlichtkrull, H., The Langlands parameters of Flensted-Jensen's discrete series for semisimple symmetrics spaces, J. Func. Anal. 50 (1983), 133-150.

[17] Schlichtkrull, H., Applications of hyperfunction Theory to representations of semisimple Lie groups. Rapport $2 a-b$, Dept. of Math., University of Copenhagen, April 1983.

[18] Strichartz, R.S., Harmonic analysis on hyperboloids. J. Funct. Anal. 12 (1973), 341-383.

[19] Takahashi, R., Quelques résultats sur l'Analyse Harmonique dans l'espace symétrique non compact de rang 1 du type exceptionnel. In: Lecture notes in Mathematics vol. 793, 511-567. Springer verlag, Berlin, 1979.

[20] Vogan, D., Algebraic structure of irreducible representations of semisimple Lie groups. Ann. of Math. 109 (1979), 1-60.

[21] Speh, B. and Vogan, D., Reducibility of generalized principal series representations. Acta Math. 145 (1980), 227-299.

[22] wolf, J.A., Spaces of constant curvature. McGraw-Hill, New York, 1967.

Mogens Flensted-Jensen Dept. Math. \& Stat. The Royal Veterinary and Agricultural University DK-1871 Copenhagen V Denmark
Kiyosato Okamoto

Dept. of Mathematics

Hiroshima University

Hiroshima

Japan 